\title{
Introduction to the Theory of Linear Operators
}

\author{
Alain Joye \\ Institut Fourier, Université de Grenoble 1, \\ BP 74, 38402 Saint-Martin d'Hères Cedex, France \\ alain.joye@ujf-grenoble.fr
}

\section{Introduction}

The purpose of this first set of lectures about Linear Operator Theory is to provide the basics regarding the mathematical key features of unbounded operators to readers that are not familiar with such technical aspects. It is a necessity to deal with such operators if one wishes to study Quantum Mechanics since such objects appear as soon as one wishes to consider, say, a free quantum particle in $\mathbb{R}$. The topics covered by these lectures is quite basic and can be found in numerous classical textbooks, some of which are listed at the end of these notes. They have been selected in order to provide the reader with the minimal background allowing to proceed to the more advanced subjects that will be treated in subsequent lectures, and also according to their relevance regarding the main subject of this school on Open Quantum Systems. Obviously, there is no claim about originality in the presented material. The reader is assumed to be familiar with the theory of bounded operators on Banach spaces and with some of the classical abstract Theorems in Functional Analysis.

\section{Generalities about Unbounded Operators}

Let us start by setting the stage, introducing the basic notions necessary to study linear operators. While we will mainly work in Hilbert spaces, we state the general definitions in Banach spaces.

If $\mathcal{B}$ is a Banach space over $\mathbb{C}$ with norm $\|\cdot\|$ and $T$ is a bounded linear operator on $\mathcal{B}$, i.e. $T: \mathcal{B} \rightarrow \mathcal{B}$, its norm is given by

$$
\|T\|=\sup _{\varphi \neq 0} \frac{\|T \varphi\|}{\|\varphi\|}<\infty .
$$

Now, consider the position operator of Quantum Mechanics $q=$ mult $x$ on $L^{2}(\mathbb{R})$, acting as $(q \varphi)(x)=x \varphi(x)$. It readily seen to be unbounded since one can find a sequence of normalized functions $\varphi_{n} \in L^{2}(\mathbb{R}), n \in \mathbb{N}$, such that $\left\|q \varphi_{n}\right\| \rightarrow \infty$ as $n \rightarrow \infty$, and, there are functions of $L^{2}(\mathbb{R})$ which are no longer $L^{2}(\mathbb{R})$ when multiplied by the independent variable. We shall adopt the following definition of (possibly unbounded) operators. 
Definition 2.1. $A$ linear operator on $\mathcal{B}$ is a pair $(A, D)$ where $D \subset \mathcal{B}$ is a dense linear subspace of $\mathcal{B}$ and $A: D \rightarrow \mathcal{B}$ is linear.

We will nevertheless often talk about the operator $A$ and call the subspace $D$ the domain of $A$. It will sometimes be denoted by $\operatorname{Dom}(A)$.

Definition 2.2. If $(\tilde{A}, \tilde{D})$ is another linear operator such that $\tilde{D} \supset D$ and $\tilde{A} \varphi=A \varphi$ for all $\varphi \in D$, the operator $\tilde{A}$ defines an extension of $A$ and one denotes this fact by $A \subset \tilde{A}$

That the precise definition of the domain of a linear operator is important for the study of its properties is shown by the following

Example 2.1. : Let $H$ be defined on $L^{2}[a, b], a<b$ finite, as the differential operator $H \varphi(x)=-\varphi^{\prime \prime}(x)$, where the prime denotes differentiation. Introduce the dense sets $D_{D}$ and $D_{N}$ in $L^{2}[a, b]$ by

$$
\begin{aligned}
& D_{D}=\left\{\varphi \in C^{2}[a, b] \mid \varphi(a)=\varphi(b)=0\right\} \\
& D_{N}=\left\{\varphi \in C^{2}[a, b] \mid \varphi^{\prime}(a)=\varphi^{\prime}(b)=0\right\} .
\end{aligned}
$$

It is easily checked that 0 is an eigenvalue of $\left(H, D_{N}\right)$ but not of $\left(H, D_{D}\right)$. The boundary conditions appearing in (1), (2) respectively are called Dirichlet and Neumann boundary conditions respectively.

The notion of continuity naturally associated with bounded linear operators is replaced for unbounded operators by that of closedness.

Definition 2.3. Let $(A, D)$ be an operator on $\mathcal{B}$. It is said to be closed if for any sequence $\varphi_{n} \in D$ such that

$$
\varphi_{n} \rightarrow \varphi \in \mathcal{B} \text { and } A \varphi_{n} \rightarrow \psi \in \mathcal{B},
$$

it follows that $\varphi \in D$ and $A \varphi=\psi$.

Remark 2.1. i) In terms of the the graph of the operator $A$, denoted by $\Gamma(A)$ and given by

$$
\Gamma(A)=\{(\varphi, \psi) \in \mathcal{B} \times \mathcal{B} \mid \varphi \in D, \psi=A \varphi\},
$$

we have the equivalence

$A$ closed $\Longleftrightarrow \Gamma(A)$ closed (for the norm $\|(\varphi, \psi)\|^{2}=\|\varphi\|^{2}+\|\psi\|^{2}$ ).

ii) If $D=\mathcal{B}$, then $A$ is closed if and only if $A$ is bounded, by the Closed Graph Theorem ${ }^{\mathrm{a}}$.

iii) If $A$ is bounded and closed, then $\bar{D}=\mathcal{B}$ so that it is possible to extend $A$ to the whole of $\mathcal{B}$ as a bounded operator.

iv) If $A: D \rightarrow D^{\prime} \subset \mathcal{B}$ is one to one and onto, then $A$ is closed is equivalent

${ }^{\text {a If } T: \mathcal{X}} \rightarrow \mathcal{Y}$, where $\mathcal{X}$ and $\mathcal{Y}$ are two Banach spaces, then $T$ is bounded iff the graph of $T$ is closed. 
to $A^{-1}: D^{\prime} \rightarrow D$ is closed. This last property can be seen by introducing the inverse graph of $A, \Gamma^{\prime}(A)=\{(x, y) \in \mathcal{B} \times \mathcal{B} \mid y \in D, x=A y\}$ and noticing that $A$ closed iff $\Gamma^{\prime}(A)$ is closed and $\Gamma(A)=\Gamma^{\prime}\left(A^{-1}\right)$.

The notion of spectrum of operators is a key issue for applications in Quantum Mechanics. Here are the relevant definitions.

Definition 2.4. The spectrum $\sigma(A)$ of an operator $(A, D)$ on $\mathcal{B}$ is defined by its complement $\sigma(A)^{C}=\rho(A)$, where the resolvent set of $A$ is given by

$$
\begin{aligned}
\rho(A)=\{z \in \mathbb{C} \mid & (A-z): D \rightarrow \mathcal{B} \text { is one to one and onto, and } \\
& \left.(A-z)^{-1}: \mathcal{B} \rightarrow D \text { is a bounded operator. }\right\}
\end{aligned}
$$

The operator $R(z)=(A-z)^{-1}$ is called the resolvent of $A$.

Actually, $A-z$ is to be understood as $A-z I I$, where II denotes the identity operator.

Here are a few of the basic properties related to these notions.

Proposition 2.1. With the notations above,

i) If $\sigma(A) \neq \mathbb{C}$, then $A$ is closed.

ii) If $z \notin \sigma(A)$ and $u \in \mathbb{C}$ is such that $|u|<\|R(z)\|^{-1}$, then $z+u \in \rho(A)$.

Thus, $\rho(A)$ is open and $\sigma(A)$ is closed.

iii) The resolvent is an analytic map from $\rho(A)$ to $\mathcal{L}(\mathcal{B})$, the set of bounded linear operators on $\mathcal{B}$, and the following identities hold for any $z, w \in \rho(A)$,

$$
\begin{aligned}
& R(z)-R(w)=(z-w) R(z) R(w) \\
& \frac{d^{n}}{d z^{n}} R(z)=n ! R^{n+1}(z) .
\end{aligned}
$$

Remark 2.2. Identity (3) is called the first resolvent identity. As a consequence, we get that the resolvents at two different points of the resolvent set commute, i.e.

$$
[R(z), R(w)]=0, \forall z, w \in \rho(A) .
$$

Proof. i) If $z \in \rho(A)$, then $R(z)$ is one to one and bounded thus closed and remark iv) above applies.

ii) We need to show that $R(z+u)$ exists and is bounded from $\mathcal{B}$ to $D$. We have on $D$

$$
(A-z-u) \varphi=\left(I I-u(A-z)^{-1}\right)(A-z) \varphi=(I I-u R(z))(A-z) \varphi,
$$

where $|u|\|R(z)\|<1$ by assumption. Hence, the Neumann series

$$
\sum_{n \geq 0} T^{n}=(I I-T)^{-1} \text { where } T: \mathcal{B} \rightarrow \mathcal{B} \text { is such that }\|T\|<1,
$$

shows that the natural candidate for $(A-z-u)^{-1}$ is $R(z)(I I-u R(z))^{-1}$ : $\mathcal{B} \rightarrow D$. Then one checks that on $\mathcal{B}$ 


$$
(A-z-u) R(z)(I I-u R(z))^{-1}=(I I-u R(z))(I I-u R(z))^{-1}=I I
$$

and that on $D$

$$
\begin{aligned}
R(z)(I I-u R(z))^{-1}(A-z-u)= & (I I-u R(z))^{-1} R(z)(A-z-u)= \\
& (I I-u R(z))^{-1}(I I-u R(z))=\Pi_{D},
\end{aligned}
$$

where $\Pi_{D}$ denotes the identity of $D$.

iii) By (4) we can write

$$
R(z+u)=\sum_{n \geq 0} u^{n} R^{n+1}(z)
$$

so that we get the analyticity of the resolvent and the expression for its derivatives. Finally for $\varphi \in D$

$$
((A-z)-(A-w)) \varphi=(w-z) \varphi
$$

so that, for any $\psi \in \mathcal{B}$,

$$
R(z)((A-z)-(A-w)) R(w) \psi=R(w) \psi-R(z) \psi=R(z) R(w)(w-z) \psi
$$

where $R(w) \psi \in D$.

Note that in the bounded case, the spectrum of an operator is never empty nor equal to $\mathbb{C}$, whereas there exist closed unbounded operators with empty spectrum or empty resolvent set. Consider for example, $T=i \frac{d}{d x}$ on $L^{2}[0,1]$ on the following dense sets. If $A C^{2}[0,1]$ denotes the set of absolutely continuous functions on $[0,1]$ whose derivatives are in $L^{2}[0,1]$, (hence in $L^{1}[0,1]$ ), set

$$
D_{1}=\left\{\varphi \mid \varphi \in A C^{2}[0,1]\right\}, \quad D_{0}=\left\{\varphi \mid \varphi \in A C^{2}[0,1] \text { and } \varphi(0)=0\right\} \text {. }
$$

Then, one checks that $\left(T, D_{1}\right)$ and $\left(T, D_{0}\right)$ are closed and such that $\sigma_{1}(T)=\mathbb{C}$ and $\sigma_{0}(T)=\emptyset$ (with the obvious notations).

To avoid potential problems related to the fact that certain operators can be a priori defined on dense sets on which they may not be closed, one introduces the following notions.

Definition 2.5. An operator $(A, D)$ is closable if it possesses a closed exten$\operatorname{sion}(\tilde{A}, \tilde{D})$.

Lemma 2.1. If $(A, D)$ is closable, then there exists a unique extension $(\bar{A}, \bar{D})$ called the closure of $(A, D)$ characterized by the fact that $\bar{A} \subseteq \tilde{A}$ for any closed extension $(\tilde{A}, \tilde{D})$ of $(A, D)$.

Proof. Let

$$
\bar{D}=\left\{\varphi \in \mathcal{B} \mid \exists \varphi_{n} \in D \text { and } \psi \in \mathcal{B} \text { with } \varphi_{n} \rightarrow \varphi \text { and } A \varphi_{n} \rightarrow \psi\right\} .
$$


For any closed extension $\tilde{A}$ of $A$ and any $\varphi \in \bar{D}$, we have $\varphi \in \tilde{D}$ and $\tilde{A} \varphi=\psi$ is uniquely determined by $\varphi$. Let us define $(\bar{A}, \bar{D})$ by $\bar{A} \varphi=\psi$, for all $\varphi \in \bar{D}$. Then $\bar{A}$ is an extension of $A$ and any closed extension $A \subseteq \tilde{A}$ is such that $\bar{A} \subseteq \tilde{A}$. The graph $\Gamma(\bar{A})$ of $\bar{A}$ satisfies $\Gamma(\bar{A})=\overline{\Gamma(A)}$, so that $\overline{\bar{A}}$ is closed.

Note also that the closure of a closed operator coincide with the operator itself. Also, before ending this section, note that there exist non closable operators. Fortunately enough, such operators do not play an essential role in Quantum Mechanics, as we will shortly see.

\section{Adjoint, Symmetric and Self-adjoint Operators}

The arena of Quantum Mechanics is a complex Hilbert space $\mathcal{H}$ where the notion of scalar product $\langle\cdot \mid \cdot\rangle$ gives rise to a norm denoted by $\|\cdot\|$. Operators that are self-adjoint with respect to that product play a particularly important role, as they correspond to the observables of the theory. We shall assume the following convention regarding the positive definite sesquilinear form $\langle\cdot \mid \cdot\rangle$ on $\mathcal{H} \times \mathcal{H}$ : it is linear in the right variable and thus anti-linear in the left variable. We shall also always assume that our Hilbert space is separable, i.e. it admits a countable basis, and we shall identify the dual $\mathcal{H}^{\prime}$ of $\mathcal{H}$ with $\mathcal{H}$, since $\forall l: \mathcal{H} \rightarrow \mathbb{C}, \exists ! \psi \in \mathcal{H}$ such that $l(\cdot)=\langle\psi \mid \cdot\rangle$.

Let us make the first steps towards self-adjunction.

Definition 3.1. An operator $(H, D)$ in $\mathcal{H}$ is said to be symmetric if $\forall \varphi, \psi \in$ $D \subseteq \mathcal{H}$

$$
\langle\varphi \mid H \psi\rangle=\langle H \varphi \mid \psi\rangle .
$$

For example, the operators $\left(-\frac{d^{2}}{d x^{2}}, D_{D}\right)$ and $\left(-\frac{d^{2}}{d x^{2}}, D_{N}\right)$ introduced above are symmetric, as shown by integration by parts.

Remark 3.1. If $H$ is symmetric, its eigenvalues are real.

The next property is related to an earlier remark concerning the role of non closable operators in Quantum Mechanics.

Proposition 3.1. Any symmetric operator $(H, D)$ is closable and its closure is symmetric.

This Proposition will allow us to consider that any symmetric operator is closed from now on.

Proof. Let $\bar{D} \supseteq D$ as in (5) and $\chi \in D, \varphi \in \bar{D}$. We compute for any such $\chi$,

$$
\langle\varphi \mid H \chi\rangle=\lim _{n}\left\langle\varphi_{n} \mid H \chi\right\rangle=\lim _{n}\left\langle H \varphi_{n} \mid \chi\right\rangle=\langle\psi \mid \chi\rangle .
$$

As $D$ is dense by assumption, the vector $\psi$ is uniquely determined by the linear, bounded form $l_{\psi}: D \rightarrow \mathbb{C}$ such that $l_{\psi}(\chi)=\langle\psi \mid \chi\rangle$. In other words, 
$\psi$ is characterized by $\varphi$ uniquely. One then defines $\bar{H}$ on $\bar{D}$ by $\bar{H} \varphi=\psi$ and linearity is easily checked. As, by construction, $\Gamma(\bar{H})=\overline{\Gamma(H)}$ is closed, $\bar{H}$ is a closed extension of $H$. Let us finally check the symmetry property. If $\chi_{n} \in D$ is such that $\chi_{n} \rightarrow \chi \in \bar{D}$, with $H \chi_{n} \rightarrow \eta$ and $\varphi \in \bar{D}$, (6) says

$$
\left\langle\varphi \mid H \chi_{n}\right\rangle=\left\langle\bar{H} \varphi \mid \chi_{n}\right\rangle \text {. }
$$

Taking the limit $n \rightarrow \infty$, we get from the above

$$
\lim _{n}\left\langle\varphi \mid H \chi_{n}\right\rangle=\langle\varphi \mid \eta\rangle=\langle\varphi \mid \bar{H} \chi\rangle=\lim _{n}\left\langle\bar{H} \varphi \mid \chi_{n}\right\rangle=\langle\bar{H} \varphi \mid \chi\rangle .
$$

When dealing with bounded operators, symmetric and self-adjoint operators are identical. It is not necessary true in the unbounded case. As one of the most powerful tools in linear operator theory, namely the Spectral Theorem, applies only to self-adjoint operators, we will develop some criteria to distinguish symmetric and self-adjoint operators.

Definition 3.2. Let $(A, D)$ be an operator on $\mathcal{H}$. The adjoint of $A$, denoted by $\left(A^{*}, D^{*}\right)$, is determined as follows: $D^{*}$ is the set of $\psi \in \mathcal{H}$ such that there exists a $\chi \in \mathcal{H}$ so that

$$
\langle\psi \mid A \varphi\rangle=\langle\chi \mid \varphi\rangle, \quad \forall \varphi \in D .
$$

As $D$ is dense, $\chi$ is unique, so that one sets $A^{*} \psi=\chi$ and checks easily the linearity. Therefore,

$$
\langle\psi \mid A \varphi\rangle=\left\langle A^{*} \psi \mid \varphi\right\rangle, \forall \varphi \in D, \psi \in D^{*} .
$$

In other words, $\psi \in D^{*}$ iff the linear form $l(\cdot)=\langle\psi \mid A \cdot\rangle: D \rightarrow \mathbb{C}$ is bounded. In that case, Riesz Lemma implies the existence of a unique $\chi$ such that $\langle\psi \mid A \cdot\rangle=\langle\chi \mid \cdot\rangle$. Note also that $D^{*}$ is not necessarily dense.

Let us proceed with some properties of the adjoint.

Proposition 3.2. Let $(A, D)$ be an operator on $\mathcal{H}$.

i) The adjoint $\left(A^{*}, D^{*}\right)$ of $(A, D)$ is closed. If, moreover, $A$ is closable, then

$D^{*}$ is dense

ii) If $A$ is closable, $\bar{A}=A^{* *}$

iii) If $A \subseteq B$, then $B^{*} \subseteq A^{*}$.

Proof. i) Let $(\psi, \chi) \in D^{*} \times \mathcal{H}$ belong to $\Gamma\left(A^{*}\right)$. This is equivalent to saying

$$
\langle\psi \mid A \varphi\rangle=\langle\chi \mid \varphi\rangle, \forall \varphi \in D,
$$

which is equivalent to $(\psi, \chi) \in M^{\perp}$, where

$$
M=\{(A \varphi,-\varphi) \in \mathcal{H} \times \mathcal{H}, \mid \varphi \in D\},
$$

with the scalar product $\left\langle\left\langle\left(\varphi_{1}, \varphi_{2}\right) \mid\left(\psi_{1}, \psi_{2}\right)\right\rangle\right\rangle=\left\langle\varphi_{1} \mid \psi_{1}\right\rangle+\left\langle\varphi_{2} \mid \psi_{2}\right\rangle$. As $M^{\perp}$ is closed, $\Gamma\left(A^{*}\right)$ is closed too. Assume now $A$ is closable and suppose there 
exists $\eta \in \mathcal{H}$ such that $\langle\psi \mid \eta\rangle=0$, for all $\psi \in D^{*}$. This implies that $(\eta, 0)$ is orthogonal to $\Gamma\left(A^{*}\right)$. But,

$$
\Gamma\left(A^{*}\right)^{\perp}=M^{\perp \perp}=\bar{M} .
$$

Therefore, there exists $\varphi_{n} \in D$, such that $\varphi_{n} \rightarrow 0$ and $A \varphi_{n} \rightarrow \eta$. As $A$ is closable, $\eta=\bar{A} 0=0$, i.e. $\left(D^{*}\right)^{\perp}=0$ and $\overline{D^{*}}=\left(D^{*}\right)^{\perp \perp}=\mathcal{H}$.

ii) Define a unitary operator $V$ on $\mathcal{H} \times \mathcal{H}$ by

$$
V(\varphi, \psi)=(\psi,-\varphi) .
$$

It has the property $V\left(E^{\perp}\right)=(V(E))^{\perp}$, for any linear subspace $E \subseteq \mathcal{H} \times \mathcal{H}$. In particular, we have just seen

$$
\Gamma\left(A^{*}\right)=(V(\Gamma(A)))^{\perp}
$$

so that

$$
\begin{aligned}
\overline{\Gamma(A)} & =\left(\Gamma(A)^{\perp}\right)^{\perp}=\left(\left(V^{2} \Gamma(A)\right)^{\perp}\right)^{\perp} \\
& =\left(V\left(V(\Gamma(A))^{\perp}\right)\right)^{\perp}=\left(V\left(\Gamma\left(A^{*}\right)\right)\right)^{\perp}=\Gamma\left(A^{* *}\right),
\end{aligned}
$$

i.e. $\bar{A}=A^{* *}$.

iii) Follows readily from the definition.

When $H$ is symmetric, we get from the definition and properties above that $H^{*}$ is a closed extension of $H$. This motivates the

Definition 3.3. An operator $(H, D)$ is self-adjoint whenever it coincides with its adjoint $\left(H^{*}, D^{*}\right)$. It is therefore closed.

An operator $(H, D)$ is essentially self-adjoint if it is symmetric and its closure $(\bar{H}, \bar{D})$ is self-adjoint.

Therefore, we have in general for a symmetric operator,

$$
H \subseteq \bar{H}=H^{* *} \subseteq H^{*}, \text { and } H^{*}=\overline{H^{*}}=H^{* * *}=\bar{H}^{*} .
$$

In case $H$ is essentially self-adjoint,

$$
H \subseteq \bar{H}=H^{* *}=H^{*} .
$$

We now head towards our general criterion for (essential) self-adjointness. We need a few more

Definition 3.4. For $(H, D)$ symmetric and denoting its adjoint by $\left(H^{*}, D^{*}\right)$, the deficiency subspaces $L^{ \pm}$are defined by

$$
\begin{aligned}
L^{ \pm} & =\left\{\varphi \in D^{*} \mid H^{*} \varphi= \pm i \varphi\right\}=\{\varphi \in \mathcal{H} \mid\langle H \psi \mid \varphi\rangle= \pm i\langle\psi \mid \varphi\rangle \forall \psi \in D\} \\
& =\operatorname{Ran}(H \pm i)^{\perp}=\operatorname{Ker}\left(H^{*} \mp i\right) .
\end{aligned}
$$


The deficiency indices are the dimensions of $L^{ \pm}$, which can be finite or infinite.

To get an understanding of these names, recall that one can always write

$$
\mathcal{H}=\operatorname{Ker}\left(H^{*} \mp i\right) \oplus \overline{\operatorname{Ran}(H \pm i)} \equiv L^{ \pm} \oplus \overline{\operatorname{Ran}(H \pm i)} .
$$

Note that the definitions of $L^{ \pm}$is invariant if one replaces $H$ by its closure $\bar{H}$.

For $(H, D)$ symmetric and any $\varphi \in D$ observe that

$$
\|(H+i) \varphi\|^{2}=\|H \varphi\|^{2}+\|\varphi\|^{2}=\|(H-i) \varphi\|^{2} \neq 0 .
$$

This calls for the next

Definition 3.5. Let $(H, D)$ be symmetric. The Cayley transform of $H$ is the isometric operator

$$
U=(H-i)(H+i)^{-1}: \operatorname{Ran}(H+i) \rightarrow \operatorname{Ran}(H-i) .
$$

It enjoys the following property.

Lemma 3.1. The symmetric extensions of $H$ are in one to one correspondence with the isometric extensions of $U$.

Proof. Let $(\tilde{H}, \tilde{D})$ be a symmetric extension of $(H, D)$ and $\tilde{U}$ be its Cayley transform. We have

$$
\varphi \in \operatorname{Ran}(H \pm i) \Longleftrightarrow \exists \psi \in D \subseteq \tilde{D} \text { such that } \varphi=(H \pm i) \psi=(\tilde{H} \pm i) \psi,
$$

hence $\operatorname{Ran}(H \pm i) \subset \operatorname{Ran}(\tilde{H} \pm i)$, and

$$
\tilde{U} \varphi=(\tilde{H}-i)(\tilde{H}+i)^{-1} \varphi=U \varphi, \quad \forall \varphi \in \operatorname{Ran}(H \pm i) .
$$

Conversely, let $\tilde{U}: M^{+} \rightarrow M^{-}$, be a isometric extension of $U$, where $\operatorname{Ran}(H \pm$ i) $\subseteq M^{ \pm}$. We need to construct a symmetric extension of $H$ whose Cayley transform is $\tilde{U}$. Algebraically this means, see (8),

$$
\tilde{H}=(\tilde{U}-I)^{-1} \frac{2}{i}-i
$$

Let us show that 1 is not an eigenvalue of $\tilde{U}$. If $\varphi \in M^{+}$is a corresponding eigenvenvector, and $\psi=(H+i) \chi$, where $\chi \in D$, then

$$
\begin{aligned}
2 i\langle\varphi \mid \chi\rangle & =\langle\varphi \mid(H+i) \chi-(H-i) \chi\rangle=\langle\varphi \mid \psi-U \psi\rangle \\
& =\langle\varphi \mid \psi\rangle-\langle\tilde{U} \varphi \mid \tilde{U} \psi\rangle=0 .
\end{aligned}
$$

By density of $D, \varphi=0$, so that we can define $\tilde{H}$ by $(9)$ on $\tilde{D}=(\tilde{U}-I I) M^{+}$. It is not difficult to check that $\tilde{H}$ is a symmetric extension of $H$.

We can now state the 
Theorem 3.1. If $(H, D)$ is symmetric on $\mathcal{H}$, there exist self-adjoint extensions of $H$ if and only if the deficiency indices are equal. Moreover, the following statements are equivalent:

1) $H$ is essentially self-adjoint

2) The deficiency indices are both zero

3) $H$ possesses exactly one self-adjoint extension.

Proof. 1) $\Rightarrow 3$ ): Let $J$ be a self-adjoint extension of $H$. Then $H \subseteq J=J^{*}$ and $J \supseteq \bar{H}$. Hence $J=J^{*} \subseteq \bar{H}^{*}=\bar{H}$, so that $J=\bar{H}$.

$1) \Rightarrow 2$ ): We can assume that $H$ is closed so $H=\bar{H}=H^{*}$. For any $\varphi \in L^{ \pm}=$ $\operatorname{Ker}\left(H^{*} \mp i\right)$,

$$
0=\left\|\left(H^{*} \mp i\right) \varphi\right\|^{2}=\|(H \mp i) \varphi\|^{2}=\|H \varphi\|^{2}+\|\varphi\|^{2} \geq\|\varphi\|^{2}, \quad L^{ \pm}=\{0\} .
$$

$2) \Rightarrow 1)$ : Consider $(H+i): D \rightarrow \operatorname{Ran}(H+i)$. By (10) above, this operator is one to one, and we can define $(H+i)^{-1}: \operatorname{Ran}(H+i) \rightarrow D$. By the same estimate it satisfies

$$
\left\|(H+i)^{-1} \psi\right\|^{2} \leq\left\|(H+i)(H+i)^{-1} \psi\right\|^{2}=\|\psi\|^{2} .
$$

As $H$ can be assumed to be closed (i.e. $H=\bar{H}$ ) and $L^{+}=\{0\}$, we get that $\operatorname{Ran}(H+i)$ is closed so that $\mathcal{H}=\operatorname{Ran}(H+i)$, due to (7). Therefore, for any $\varphi \in D^{*}$, there exists a $\psi \in D$ such that $\left(H^{*}+i\right) \varphi=(H+i) \psi$. As $H \subseteq H^{*}$,

$$
\left(H^{*}+i\right)(\varphi-\psi)=0 \text {, i.e. } \varphi-\psi \in \operatorname{Ker}\left(H^{*}+i\right)=\{0\},
$$

we get that $\varphi \in D$ and $H=H^{*}$, which is what we set out to prove.

$3) \Rightarrow 2$ ): if $K$ is a self-adjoint extension of $H$, its deficiency indices are zero (by 2)). Therefore, (see (7)), its Cayley transform $V$ is a unitary extension of $U$, the Cayley transform of $H$. In particular, $\left.V\right|_{L^{+}}: L^{+} \rightarrow L^{-}$is one to one and onto, so that the deficiency indices of $H$ are equal. That yields the first part of the Theorem. Now assume these indices are not zero. By the preceding Lemma, there exist an infinite number of symmetric extensions of $H$, parametrized by all isometries from $L^{+}$to $L^{-}$. In particular, there exist extensions with zero deficiency indices, which by 2) and 1) are self-adjoint, contradicting the fact that $K$ is the unique self-adjoint extension of $H$.

Remark 3.2. It is a good exercise to prove that in case $(H, D)$ is symmetric and $H \geq 0$, i.e. $\langle\varphi \mid H \varphi\rangle \geq 0$ for any $\varphi \in D$, then $H$ is essentially self-adjoint iff $\operatorname{Ker}\left(H^{*}+1\right)=\{0\}$.

As a first application, we give a key property of self-adjoint operators for the role they play in the Quantum dogma concerning measure of observables. It is the following fact concerning their spectrum.

Theorem 3.2. Let $H=H^{*}$. Then, $\sigma(H) \subseteq \mathbb{R}$ and,

$$
\left\|(H-z)^{-1}\right\| \leq \frac{1}{|\Im z|}, \text { if } z \notin \mathbb{R} .
$$


Moreover, for any $z$ in the resolvent set of $H$,

$$
(H-\bar{z})^{-1}=\left((H-z)^{-1}\right)^{*} .
$$

Proof. Let $\varphi \in D, D$ being the domain of $H$ and $z=x+i y$, with $y \neq 0$. Then

$$
\|(H-x-i y) \varphi\|^{2}=\|(H-x) \varphi\|^{2}+y^{2}\|\varphi\|^{2} \geq y^{2}\|\varphi\|^{2} .
$$

This implies

$$
\operatorname{Ker}(H-z)=\operatorname{Ker}\left(H^{*}-z\right)=\{0\} \text { i.e. } \overline{\operatorname{Ran}(H-z)}=\mathcal{H},
$$

and $H-z$ is invertible on $\operatorname{Ran}(H-z)$. (13) shows that $(H-z)$ is bounded with the required bound, and as the resolvent is closed, it can be extended on $\mathcal{H}$ with the same bound. Equality (12) is readily checked.

As an application of the first part of Theorem 3.1, consider a symmetric operator $(H, D)$ which commutes with a conjugation $C$. More precisely:

$C$ is anti-linear, $C^{2}=I I$ and $\|C \varphi\|=\|\varphi\|$. Hence $\langle\varphi \mid \psi\rangle=\langle C \psi \mid C \varphi\rangle$. Moreover, $C: D \rightarrow D$ and $C H=H C$ on $D$.

Under such circumstances, the deficiency indices of $H$ are equal and there exist self-adjoint extensions of $H$.

Indeed, one first deduces that $C(D)=D$. Then, for any $\varphi^{+} \in L^{+}=$ $\operatorname{Ker}\left(H^{*}-i\right)$ and $\psi \in D$, we compute

$$
0=\overline{\left\langle\varphi^{+} \mid(H+i) \psi\right\rangle}=\left\langle C \varphi^{+} \mid C(H+i) \psi\right\rangle=\left\langle C \varphi^{+} \mid(H-i) C \psi\right\rangle,
$$

so that $C \varphi^{+} \in \operatorname{Ran}(H-i)^{\perp}=\operatorname{Ker}\left(H^{*}+i\right)=L^{-}$. In other words, $C: L^{+} \rightarrow L^{-}$, and one shows similarly that $C: L^{-} \rightarrow L^{+}$. As $C$ is isometric, the dimensions of $L^{+}$and $L^{-}$are the same.

A particular case where this happens is that of the complex conjugation and a differential operator on $\mathbb{R}^{n}$, with real valued coefficients.

An example of direct application of this criterion is the following. Consider the symmetric operator $H \varphi=i \varphi^{\prime}$ on the domain $C_{0}^{\infty}(0, \infty) \subset L^{2}(0, \infty)$. A vector $\psi \in D^{*}$ iff there exists $\chi \in L^{2}(0, \infty)$ such that $\langle\psi \mid H \varphi\rangle=\langle\chi \mid \varphi\rangle$, for all $\varphi \in C_{0}^{\infty}(0, \infty)$. Expressing the scalar products this means

$$
\int \chi(x) \bar{\varphi}(x) d x=-i \int \psi(x) \bar{\varphi}^{\prime}(x) d x=i D_{x} T_{\psi}(\bar{\varphi}),
$$

where $T_{\psi}$ denotes the distribution associated with $\psi$. In other words, we have $\psi \in W^{1,2}(0, \infty)=D^{*}$ and $H^{*} \psi=i \psi$ in the weak sense. Elements of $\operatorname{Ker}\left(H^{*} \mp i\right)$ satisfy 


$$
H^{*} \psi= \pm i \psi \Longleftrightarrow \psi^{\prime}= \pm \psi \Longleftrightarrow \psi(x)=c e^{ \pm x}\left\{\begin{array}{l}
\notin L^{2}(0, \infty) \\
\in L^{2}(0, \infty)
\end{array}\right.
$$

Hence there is no self-adjoint extension of that operator. If it is considered on $C_{0}^{\infty}(0,1) \subset L^{2}(0,1)$, the above shows that the deficiency indices are both 1 and there exist infinitely many self-adjoint extensions of it.

Specializing a little, we get a criterion for operators whose spectrum consists of eigenvalues only.

Corollary 3.1. Let $(H, D)$ symmetric on $\mathcal{H}$ such that there exists an orthonormal basis $\left\{\varphi_{n}\right\}_{n \in \mathbb{N}}$ of $\mathcal{H}$ of eigenvectors of $H$ satisfying for any $n \in \mathbb{N}$, $\varphi_{n} \in D$ and $H \varphi_{n}=\lambda_{n} \varphi_{n}$, with $\lambda_{n} \in \mathbb{R}$. Then $H$ is essentially self-adjoint and $\sigma(\bar{H})=\overline{\left\{\lambda_{n} \mid n \in \mathbb{N}\right\}}$.

Proof. Just note that any vector $\varphi$ in $L^{ \pm}$satisfies in particular

$$
\left\langle H \varphi_{n} \mid \varphi\right\rangle= \pm i\left\langle\varphi_{n} \mid \varphi\right\rangle=\lambda_{n}\left\langle\varphi_{n} \mid \varphi\right\rangle
$$

so that $\left\langle\varphi_{n} \mid \varphi\right\rangle=0$ for any $n$. This means that $L^{+}=\{0\}$, hence that $H$ is essentially self-adjoint.

Then $\bar{H}$, as an extension of $H$ admits the $\varphi_{n}$ 's as eigenvectors with the same eigenvalues and as the spectrum is a closed set, we get $\sigma(\bar{H}) \supset$ $\overline{\left\{\lambda_{n} \mid n \in \mathbb{N}\right\}}$. If $\lambda$ does not belong to the latter set, we define $R_{\lambda}$ by $R_{\lambda} \varphi_{n}=$ $\frac{1}{\lambda_{n}-\lambda} \varphi_{n}$, for all $n \in \mathbb{N}$. Using the fact that $\bar{H}$ is closed, it is not difficult to see that $R_{\lambda}$ is the resolvent of $\bar{H}$ at $\lambda$, which yields the result.

As a first example of application we get that $-\frac{d^{2}}{d x^{2}}$ on $C^{2}(a, b)$ (or $\left.C^{\infty}(a, b)\right)$ with Dirichlet boundary conditions is essentially self-adjoint with spectrum $\left\{\frac{n^{2} \pi^{2}}{(b-a)^{2}}\right\}_{n \in \mathbb{N}^{*}}$, as the corresponding eigenvectors

$$
\varphi_{n}(x)=\left(\frac{2}{b-a}\right)^{1 / 2} \sin \left(\frac{n \pi(x-a)}{b-a}\right), \quad n \in \mathbb{N}^{*},
$$

are known to form a basis of $L^{2}[a, b]$ by the theory of Fourier series.

Another standard operator is the harmonic oscillator defined on $L^{2}(\mathbb{R})$ by the differential operator

$$
H_{o s c}=-\frac{1}{2} \frac{d^{2}}{d x^{2}}+\frac{x^{2}}{2}
$$

with dense domain $\mathcal{S}$ the Schwartz functions. This operator is symmetric by integration by parts, and it is a standard exercise, using creation and annihilation operators $b^{\dagger}=\left(x-\partial_{x}\right) / \sqrt{2}, b=\left(x+\partial_{x}\right) / \sqrt{2}$ to show that the solutions of

$$
H_{o s c} \varphi_{n}(x)=\lambda_{n} \varphi_{n}(x), \quad n \in \mathbb{N},
$$

are given by $\lambda_{n}=n+1 / 2$ with eigenvector 


$$
\varphi_{n}(x)=c_{n} H_{n}(x) e^{-x^{2} / 2}, \text { with } H_{n}(x)=(-1)^{n} e^{x^{2}} \frac{d^{n}}{d x^{n}} e^{-x^{2}},
$$

and $c_{n}=\left(2^{n} n ! \sqrt{\pi}\right)^{-1 / 2}$. These eigenvectors also form a basis of $L^{2}(\mathbb{R})$, so that this operator is essentially self-adjoint with spectrum $\mathbb{N}+1 / 2$. Note that cannot work on $C_{0}^{\infty}$ to apply this criterion here.

Another popular way to prove that an operator is self-adjoint is to compare it to another operator known to be self-adjoint and use a perturbative argument to get self-adjointness of the former.

Let $(H, D)$ be a self-adjoint operator on $\mathcal{H}$ and let $(A, D(A))$ be symmetric with domain $D(A) \supseteq D$.

Definition 3.6. The operator $A$ has a relative bound $\alpha \geq 0$ with respect to $H$ if there exists $c<\infty$ such that

$$
\|A \varphi\| \leq \alpha\|H \varphi\|+c\|\varphi\|, \forall \varphi \in D .
$$

The infimum over such relative bounds is the relative bound of $A$ w.r.t. $H$.

Remark 3.3. The definition of the relative bound is unchanged if we replace (14) by the slightly stronger condition

$$
\|A \varphi\|^{2} \leq \alpha^{2}\|H \varphi\|^{2}+c^{2}\|\varphi\|^{2}, \forall \varphi \in D .
$$

Lemma 3.2. Let $K: D \rightarrow \mathcal{H}$ be such that $K \varphi=H \varphi+A \varphi$. If $0 \leq \alpha<1$, is the relative bound of $A$ w.r.t. $H, K$ is closed and symmetric. Moreover, $\left\|A(H+i \lambda)^{-1}\right\|<1$, if $\lambda \in \mathbb{R}$ has large enough modulus.

Proof. The symmetry of $K$ is clear. Let us consider $\varphi_{n} \in D$ such that $\varphi_{n} \rightarrow \varphi$ and $K \varphi_{n} \rightarrow \psi$. Then, by assumption,

$$
\begin{aligned}
\left\|H \varphi_{n}-H \varphi_{m}\right\| & \leq\left\|K \varphi_{n}-K \varphi_{m}\right\|+\left\|A \varphi_{n}-A \varphi_{m}\right\| \\
& \leq\left\|K \varphi_{n}-K \varphi_{m}\right\|+\alpha\left\|H \varphi_{n}-H \varphi_{m}\right\|+c\left\|\varphi_{n}-\varphi_{m}\right\|,
\end{aligned}
$$

so that

$\left\|H \varphi_{n}-H \varphi_{m}\right\| \leq \frac{1}{1-\alpha}\left\|K \varphi_{n}-K \varphi_{m}\right\|+\frac{c}{1-\alpha}\left\|\varphi_{n}-\varphi_{m}\right\| \rightarrow 0$ as $n, m \rightarrow \infty$.

$H$ being closed, we deduce from the above that $\varphi \in D$ and $H \varphi_{n} \rightarrow H \varphi$. Then, from (14), we get $A \varphi_{n}-A \varphi \rightarrow 0$ from which follows $K \varphi_{n} \rightarrow K \varphi=\psi$.

The proof of the statement concerning the resolvent reads as follows. Let $\psi \in \mathcal{H}, \varphi=(H+i \lambda)^{-1} \psi$ and $0 \leq \alpha<\beta<1$. Then, for $|\lambda|>0$ large enough

$$
\begin{aligned}
\|A \varphi\|^{2} & \leq(\alpha\|H \varphi\|+c\|\varphi\|)^{2} \leq \beta^{2}\left(\|H \varphi\|^{2}+\lambda^{2}\|\varphi\|^{2}\right) \\
& =\beta^{2}\|(H+i \lambda) \varphi\|^{2}=\beta^{2}\|\psi\|^{2} .
\end{aligned}
$$

Hence $\left\|A(H+i \lambda)^{-1} \psi\right\| \leq \beta\|\psi\|$.

This leads to the 
Theorem 3.3. If $H$ is self-adjoint and $A$ is symmetric with relative bound $\alpha<1$ w.r.t. $H$, then $K=H+A$ is self-adjoint on the same domain as that of $H$.

Proof. Let $|\lambda|$ be large enough. From the formal expressions

$$
\begin{aligned}
& (H+A+i \lambda)^{-1}-(H+i \lambda)^{-1}=-(H+A+i \lambda)^{-1}(A)(H+i \lambda)^{-1} \Longleftrightarrow \\
& (H+A+i \lambda)^{-1}=(H+i \lambda)^{-1}\left(I I+A(H+i \lambda)^{-1}\right)^{-1}
\end{aligned}
$$

we see that the natural candidate for the resolvent of $K$ is

$$
R_{\lambda}=(H+i \lambda)^{-1} \sum_{n \in \mathbb{N}}\left(-A(H+i \lambda)^{-1}\right)^{n} .
$$

By assumption on $|\lambda|$, this sum converges in norm and $\operatorname{Ran}\left(R_{\lambda}\right)=D$. Routine manipulations show that $(H+A+i \lambda) R_{\lambda}=R_{\lambda}(H+A+i \lambda)=I I$ so that $\operatorname{Ran}(H+A+i \lambda)=\mathcal{H}$. This implies that the deficiency indices of $K=H+A$ are both zero, and since it is closed, $K$ is self-adjoint. Note that one uses the fact that $\operatorname{dim} \operatorname{ker}\left(K^{*}-i \lambda\right)$ is constant for $\lambda>0$ and $\lambda<0$.

\section{Spectral Theorem}

Let us start this section by the presentation of another example of self-adjoint operator, which will play a key role in the Spectral Theorem, we set out to prove here. Before getting to work, let us specify right away that we shall not provide here a full proof of the version of the Spectral Theorem we chose. Some parts of it, of a purely analytical character, will be presented as facts whose detailed full proofs can be found in Davies's book [D]. But we hope to convey the main ideas of the proof in these notes.

Consider $E \subseteq \mathbb{R}^{N}$ a Borel set and $\mu$ a Borel non-negative measure on $E$. Let $\mathcal{H}=L^{2}(E, d \mu)$ be the usual set of measurable functions $f: E \rightarrow \mathbb{C}$ such that $\|f\|^{2}=\int_{E}|f(x)|^{2} d \mu<\infty$, with identification of functions that coincide almost everywhere.

Let $a: E \rightarrow \mathbb{R}$ be measurable and such that the restriction of $a$ to any bounded set of $E$ is bounded. We set

$$
D=\left\{\left.f \in \mathcal{H}\left|\int_{E}\left(1+a^{2}(x)\right)\right| f(x)\right|^{2} d \mu<\infty\right\},
$$

which is dense, and we define the multiplication operator $(A, D)$ by

$$
(A f)(x)=a(x) f(x), \quad \forall f \in D .
$$

Lemma 4.1. $(A, D)$ is self-adjoint and if $L_{c}^{2}$ denotes the set of functions of $\mathcal{H}$ which are zero outside a compact subset of $E$, then $A$ is essentially self-adjoint on $L_{c}^{2}$. 
Proof. $A$ is clearly symmetric. If $z \notin \mathbb{R}$, the bounded operator $R(z)$ given by

$$
(R(z) f)(x)=(a(x)-z)^{-1} f(x)
$$

is easily seen to be the inverse of $(A-z)$. Hence, $\sigma(A) \neq \mathbb{C}$, so that $A$ is closed. Moreover, the deficiency indices of $A$ are both seen to be zero:

$$
\begin{aligned}
A^{*} f=i f & \Leftrightarrow \forall h \in D \int \overline{A h} f d \mu=i \int \bar{h} f d \mu \\
& \Leftrightarrow \int(a(x)-i) \bar{h} f d \mu=0, \\
& \Leftrightarrow f=0 \quad \mu \text { a.e. }
\end{aligned}
$$

So that $A$ is closed and essentially self-adjoint, hence self-adjoint.

Concerning the last statement, we need to show that $A$ is the closure of its restriction to $L_{c}^{2}$. If $f \in D$ and $n \in \mathbb{N}$, we define

$$
f_{n}(x)=\left\{\begin{array}{cc}
f(x) & \text { if } x \in E,|x| \leq n \\
0 & \text { otherwise }
\end{array}\right.
$$

Hence $\left|f_{n}(x)\right| \leq|f(x)|$ and $f_{n} \in L_{c}^{2} \in D$. By Lebesgue dominated convergence Theorem, one checks that $f_{n} \rightarrow f$ and $A f_{n} \rightarrow A f$ as $n \rightarrow \infty$.

Lemma 4.2. The spectrum and resolvent of $A$ are such that

$$
\sigma(A)=\text { essential range of } a=\{\lambda \in \mathbb{R} \mid \mu(\{x|| a(x)-\lambda \mid<\epsilon\})>0, \forall \epsilon>0\} \text {. }
$$

If $\lambda \notin \sigma(A)$, then

$$
\left\|(A-\lambda)^{-1}\right\|=\frac{1}{\operatorname{dist}(\lambda, \sigma(A))} .
$$

Proof. If $\lambda$ is not in the essential range of $a$, it is readily checked that the multiplication operator by $(a(x)-\lambda)^{-1}$ is bounded (outside of a set of zero $\mu$ measure). Also one sees that this operator yields the inverse of $a-\lambda$ for such $\lambda$ 's, which, consequently, belong to $\rho(A)$. Conversely, let us take $\lambda$ in the essential range of $a$ and show that $\lambda \in \sigma(A)$. We define sets of positive $\mu$ measures by

$$
S_{m}=\left\{x \in E|| \lambda-a(x) \mid<2^{-m}\right\} .
$$

Let $\chi_{m}$ be the characteristic function of $S_{m}$, which is a non zero element of $L^{2}(E, d \mu)$. Then

$\left\|(A-\lambda) \chi_{m}\right\|^{2}=\int_{S_{m}}\left|\chi_{m}\right|^{2}|a(x)-\lambda|^{2} d \mu \leq 2^{-2 m} \int_{S_{m}}\left|\chi_{m}\right|^{2} d \mu=2^{-2 m}\left\|\chi_{m}\right\|^{2}$,

which shows that $(A-\lambda)^{-1}$ cannot be bounded. Finally, if $\lambda$ is not in the essential range of $a$, we set

$$
\left\|(a(\cdot)-\lambda)^{-1}\right\|_{\infty}=\text { essential supremum of }(a(\cdot)-\lambda)^{-1},
$$


where we recall that for a measurable function $f$

$$
\|f\|_{\infty}=\inf \{K>0|| f(x) \mid \leq K \mu \text { a.e. }\} .
$$

We immediately get that $\|a(\cdot)-\lambda\|_{\infty}$ is an upper bound for the norm of the resolvent, as, for any $\epsilon>0$ there exists a set $S \subset E$ of positive measure such that $\left|(a(x)-\lambda)^{-1}\right| \geq K-\epsilon, \forall x \in S$. Considering the characteristic function of this set, one sees that the upper bound is actually reached and corresponds with the distance of $\lambda$ to the spectrum of $A$.

\subsection{Functional Calculus}

Let us now come to the steps leading to the Spectral Theorem. The general setting is as follows. One has a self-adjoint operator $(H, D), D$ dense in a separable Hilbert space $\mathcal{H}$. We first want to define a functional calculus, allowing us to take functions of self-adjoint operators. If $H$ is a multiplication operator by a real valued function $h$, as in the above example, then $f(H)$, for a reasonable function $f: \mathbb{R} \rightarrow \mathbb{C}$, is easily conceivable as the multiplication by $f \circ h$. We are going to define a function of an operator $H$ in a quite general setting by means of an explicit formula due to Helffer and Sjöstrand and we will check that this formula has the properties we expect of such an operation. Finally, we will also see that any operator can be seen as a multiplication operator on some $L^{2}(d \mu)$ space.

Let us introduce the notation $\langle z\rangle=\left(1+|z|^{2}\right)^{1 / 2}$ and the set of functions we will work with. Let $\beta \in \mathbb{R}$ and $S^{\beta}$ be the set of complex valued $C^{\infty}(\mathbb{R})$ functions such that there exists a $c_{n}$ so that

$$
\left|f^{(n)}(x)\right|=\left|\frac{d^{n}}{d x^{n}} f(x)\right| \leq c_{n}<x>^{\beta-n}, \quad \forall x \in \mathbb{R} \forall n \in \mathbb{N} .
$$

We set $\mathcal{A}=\cup_{\beta<0} S^{\beta}$ and we define norms $\|\cdot\|_{n}$ on $\mathcal{A}$, for any $n \geq 1$, by

$$
\|f\|_{n}=\sum_{r=0}^{n} \int_{-\infty}^{\infty}\left|f^{(r)}(x)\right|<x>^{r-1} d x .
$$

This set of functions enjoys the following properties:

$\mathcal{A}$ is an algebra for the multiplication of functions, it contains the rational functions which decay to zero at $\infty$ and have non-vanishing denominator on the real axis.

Moreover, it is not difficult to see that

$$
\|f\|_{n}<\infty \Rightarrow f^{\prime} \in L^{1}(\mathbb{R}), \text { and } f(x) \rightarrow 0 \text { as }|x| \rightarrow \infty
$$

and that

$$
\left\|f-f_{k}\right\|_{n} \rightarrow 0, \text { as } k \rightarrow \infty \Rightarrow \sup _{x \in \mathbb{R}}\left|f(x)-f_{k}(x)\right| \rightarrow 0, \text { as } k \rightarrow \infty .
$$


Definition 4.1. A map which to any $f \in \mathcal{E} \subset L^{\infty}(\mathbb{R})$ associates $f(H) \in$ $\mathcal{L}(\mathcal{H})$ is a functional calculus if the following properties are true.

1. $f \mapsto f(H)$ is linear and multiplicative, (i.e. $f g \mapsto f(H) g(H)$ )

2. $\bar{f}(H)=(f(H))^{*}, \forall f \in \mathcal{E}$

3. $\|f(H)\| \leq\|f\|_{\infty}, \forall f \in \mathcal{E}$

4. If $w \notin \mathbb{R}$ and $r_{w}(x)=(x-w)^{-1}$, then $r_{w}(H)=(H-w)^{-1}$

5. If $f \in C_{0}^{\infty}(\mathbb{R})$ such that $\operatorname{supp}(f) \cap \sigma(H)=\emptyset$ then $f(H)=0$.

For $f \in C^{\infty}$, we define its quasi-analytic extension $\tilde{f}: \mathbb{C} \rightarrow \mathbb{C}$ by

$$
\tilde{f}(z)=\left(\sum_{r=0}^{n} f^{(r)}(x) \frac{(i y)^{r}}{r !}\right) \sigma(x, y)
$$

with $z=x+i y, n \geq 1, \sigma(x, y)=\tau(y /<x>)$, where $\tau \in C_{0}^{\infty}$ is equal to one on $[-1,1]$, has support in $[-2,2]$. We are naturally abusing notations as $\tilde{f}$ is not analytic in general, but it is $C^{\infty}$. Its support is confined to the set $|y| \leq 2<x>$ due to the presence of $\tau$. Also, the projection on the $x$ axis of the support of $\tilde{f}$ is equal to the support of $f$. The choice of $\tau$ and $n$ will turn out to have no importance for us.

Explicit computations yield

$$
\begin{aligned}
& \frac{\partial}{\partial \bar{z}} \tilde{f}(z)=\frac{1}{2}\left(\frac{\partial}{\partial x}+i \frac{\partial}{\partial y}\right) \tilde{f}(z)= \\
& \quad\left(\sum_{r=0}^{n} f^{(r)}(x) \frac{(i y)^{r}}{r !}\right) \frac{\left(\sigma_{x}(x, y)+i \sigma_{y}(x, y)\right)}{2}+f^{(n+1)}(x) \frac{(i y)^{n}}{n !} \frac{\sigma(x, y)}{2} .
\end{aligned}
$$

As $\operatorname{supp}\left(\sigma_{x}(x, y)\right)$ and $\operatorname{supp}\left(\sigma_{y}(x, y)\right)$ are included in $\operatorname{supp}\left(\tau^{\prime}(y /<x>)\right.$, i.e. in the set $\langle x\rangle \leq|y| \leq 2\langle x\rangle$, if $x$ is fixed and $y \rightarrow 0$,

$$
\left|\frac{\partial}{\partial \bar{z}} \tilde{f}(z)\right|=O\left(|y|^{n}\right)
$$

which justifies the name quasi-analytic extension as $y$ goes to zero.

Definition 4.2. For any $f \in \mathcal{A}$ and any self-adjoint operator $H$ on $\mathcal{H}$ the Helffer-Sjöstrand formula for $f(H)$ reads

$$
f(H)=\frac{1}{\pi} \int_{\mathbb{C}} \frac{\partial}{\partial \bar{z}} \tilde{f}(z)(H-z)^{-1} d x d y \in \mathcal{L}(\mathcal{H}) .
$$

Remark 4.1. This formula allows to compute functions of operators by means of their resolvent only. Therefore it holds for bounded as well as unbounded operators. Moreover, being explicit, it can yield useful bounds in concrete cases. Note also that it is linear in $f$.

We need to describe a little bit more in what sense this integral holds. 
Lemma 4.3. The expression (16) converges in norm and the following bound holds

$$
\|f(H)\| \leq c_{n}\|f\|_{n+1}, \quad \forall f \in \mathcal{A} \text { and } n \geq 1 .
$$

Proof. The integrand is bounded and $C^{\infty}$ on $\mathbb{C} \backslash \mathbb{R}$, therefore (16) converges in norm as a limit of Riemann sums on any compact of $\mathbb{C} \backslash \mathbb{R}$. It remains to deal with the limit when these sets tend to the whole of $\mathbb{C}$. Let us introduce the sets

$$
\begin{aligned}
& U=\{(x, y)|<x>\leq| y \mid \leq 2<x>\} \supseteq \operatorname{supp} \tau^{\prime}(y /<x>) \\
& V=\{(x, y)|0 \leq| y \mid \leq 2<x>\} \supseteq \operatorname{supp} \tau(y /<x>) .
\end{aligned}
$$

We easily get by explicit computations that

$$
\left|\sigma_{x}(x, y)+i \sigma_{y}(x, y)\right| \leq \frac{c \chi_{U}(x, y)}{<x>},
$$

where $\chi_{U}$ is the characteristic function of the set $U$. Using the bound (11) on the resolvent, (15), and the fact that $|y| \simeq<x>$ on $U$, we can bound the integrand of (16) by a constant times

$$
\sum_{r=0}^{n}\left|f^{(r)}(x)\right|<x>^{r-2} \chi_{U}(x, y)+\left|f^{(n+1)}(x)\right||y|^{n-1} \chi_{V}(x, y) .
$$

After integration on $y$ at fixed $x$, the integrand of the remaining integral in $x$ is bounded by a constant times

$$
\sum_{r=0}^{n}\left|f^{(r)}(x)\right|<x>^{r-1}+\left|f^{(n+1)}(x)\right|<x>^{n},
$$

hence the announced bound.

We need a few more properties regarding formula (16) before we can show it defines a functional calculus.

It is sometimes easier to deal with $C_{0}^{\infty}$ functions rather then with functions of $\mathcal{A}$. The following Lemma shows this is harmless.

Lemma 4.4. $C_{0}^{\infty}(\mathbb{R})$ is dense in $\mathcal{A}$ for the norms $\|\cdot\|_{n}$.

Proof. We use the classical technique of mollifiers. Let $\Phi \geq 0$ be smooth with the same conditions of support as $\tau$. Set $\Phi_{m}(x)=\Phi(x / m)$ for all $x \in \mathbb{R}$ and $f_{m}=\Phi_{m} f$. Hence, $f_{m} \in \mathcal{A}$ and support considerations yield

$$
\begin{aligned}
\left\|f-f_{m}\right\|_{n+1} & =\sum_{r=0}^{n+1} \int_{\mathbb{R}}\left|\frac{d^{r}}{d x^{r}}\left(f(x)\left(1-\Phi_{m}(x)\right)\right)\right|<x>^{r-1} d x \\
& \leq c_{n} \sum_{r=0}^{n+1} \int_{|x|>m}\left|f^{(r)}(x)\right|<x>^{r-1} d x \rightarrow 0, \text { as } m \rightarrow \infty .
\end{aligned}
$$


The next Lemma will be useful several times in the sequel.

Lemma 4.5. If $F \in C_{0}^{\infty}(\mathbb{C})$ and $F(z)=O\left(y^{2}\right)$ as $y \rightarrow 0$ at fixed real $x$, then

$$
\frac{1}{\pi} \int_{\mathbb{C}} \frac{\partial}{\partial \bar{z}} F(z)(H-z)^{-1} d x d y=0
$$

Proof. Suppose $\operatorname{supp} F \subset\{|x|<N,|y|<N\}$ and let $\Omega_{\delta}, \delta>0$ small such that $\Omega_{\delta} \subset\{|x|<N, \delta<|y|<N\}$. We want to apply Stokes Theorem to the above integral. Recall that

$$
\begin{aligned}
& \frac{\partial}{\partial \bar{z}}=\frac{1}{2}\left(\frac{\partial}{\partial x}+i \frac{\partial}{\partial y}\right), \frac{\partial}{\partial z}=\frac{1}{2}\left(\frac{\partial}{\partial x}-i \frac{\partial}{\partial y}\right) \\
& \Longleftrightarrow d \bar{z}=d x-i d y, d z=d x+i d y
\end{aligned}
$$

so that $d \bar{z} \wedge d z=2 i d x \wedge d y=2 i d x d y$. Moreover, since $\frac{\partial}{\partial \bar{z}}(H-z)^{-1}=0$ by analyticity,

$$
\begin{aligned}
d\left(F(z)(H-z)^{-1} d z\right) & =\frac{\partial}{\partial z}\left(F(z)(H-z)^{-1}\right) d z \wedge d z \\
& +\frac{\partial}{\partial \bar{z}}\left(F(z)(H-z)^{-1}\right) d \bar{z} \wedge d z \\
& =\frac{\partial F}{\partial \bar{z}}(z)(H-z)^{-1} d \bar{z} \wedge d z
\end{aligned}
$$

Therefore, if $I$ denotes (18), we get by Stokes Theorem

$$
\begin{aligned}
I & =\lim _{\delta \rightarrow 0} \frac{1}{2 \pi i} \int_{\Omega_{\delta}} d\left(F(z)(H-z)^{-1} d z\right)=\lim _{\delta \rightarrow 0} \frac{1}{2 \pi i} \int_{\partial \Omega_{\delta}} F(z)(H-z)^{-1} d z \\
& =\lim _{\delta \rightarrow 0} \frac{1}{2 \pi i} \int_{\substack{y=\delta \\
y=-\delta \\
|x|<N}} F(z)(H-z)^{-1} d z .
\end{aligned}
$$

Hence the bound

$$
|I| \leq \lim _{\delta \rightarrow 0} \frac{1}{2 \pi} \int_{-N}^{N}\left(\mid F\left(x+i \delta|+| F(x-i \delta \mid) \frac{1}{\delta} d x=\lim _{\delta \rightarrow 0} O(\delta)=0,\right.\right.
$$

where we used Taylor's formula $F(x, y)=\frac{y^{2}}{2} F_{y y}(x, \theta(y, x) y)$ with $\theta(x, y) \in$ $(0,1)$, so that $|F(x, y)| \leq c(x) y^{2}$.

Remark 4.2. It follows from the above proof that if $f$ has compact support, we can write

$$
f(H)=\lim _{\delta \rightarrow 0} \frac{1}{2 \pi i} \int_{\partial \Omega_{\delta}} \tilde{f}(z)(H-z)^{-1} d z .
$$

Neglecting support considerations, if $\tilde{f}$ was analytic, this is the way we would naturally define $f(H)$.

We can now show a comforting fact about our definition (16) of $f(H)$. 
Lemma 4.6. If $f \in \mathcal{A}$ and $n \geq 1$, then $f(H)$ is independent of $\sigma$ and $n$.

Proof. By density of $C_{0}^{\infty}$ in $\mathcal{A}$ for the norms $\|\cdot\|_{n}$ and Lemma 4.3, we can assume $f \in C_{0}^{\infty}$. Let $\tilde{f}_{\sigma_{1}, n}$ and $\tilde{f}_{\sigma_{2}, n}$ be associated with $\sigma_{1}$ and $\sigma_{2}$. Then

$$
\tilde{f}_{\sigma_{1}, n}-\tilde{f}_{\sigma_{2}, n}=\left(\sum_{r=0}^{n} f^{(r)}(x) \frac{(i y)^{r}}{r !}\right)\left(\tau_{1}(y /<x>)-\tau_{2}(y /<x>)\right),
$$

is identically zero for $y$ small enough, so Lemma 4.5 applies. Similarly, if $m>n \geq 1$, with similar notations,

$$
\tilde{f}_{\sigma, m}-\tilde{f}_{\sigma, n}=\sum_{r=n+1}^{m} f^{(r)}(x) \frac{(i y)^{r}}{r !} \sigma(x, y)=O\left(y^{2}\right), \text { as } \quad y \rightarrow 0, x \text { fixed }
$$

and Lemma 4.5 applies again.

We are now in a position to show that formula (16) possesses the properties of a functional calculus.

Proposition 4.1. With the notations above,

a) If $f \in C_{0}^{\infty}$ and $\operatorname{supp}(f) \cap \sigma(H)=\emptyset$, then $f(H)=0$.

b) $(f g)(H)=f(H) g(H)$, for all $f, g \in \mathcal{A}$.

c) $\bar{f}(H)=f(H)^{*}$ and $\|f(H)\| \leq\|f\|_{\infty}$.

d) $r_{w}(H)=(H-w)^{-1}, w \notin \mathbb{R}$.

Proof. a) In that case, since the compact set $\operatorname{supp}(f)$ and the closed set $\sigma(H)$ are disjoint, we can consider a finite number of contours $\gamma_{1}, \cdots, \gamma_{r}$ surrounding a region $W$ disjoint from $\sigma(H)$ containing the support of $\tilde{f}$. By Stokes Theorem again

$$
f(H)=\frac{1}{\pi} \int_{\mathbb{C}} \frac{\partial}{\partial \bar{z}} \tilde{f}(z)(H-z)^{-1} d x d y=\frac{1}{2 \pi i} \sum_{j=1}^{r} \int_{\gamma_{j}} \tilde{f}(z)(H-z)^{-1} d z \equiv 0,
$$

by our choice of $\gamma_{j}$.

b) Assume first $f, g \in C_{0}^{\infty}$, so that $K=\operatorname{supp}(\tilde{f})$ and $L=\operatorname{supp}(\tilde{g})$ are compact.

$$
\begin{aligned}
f(H) g(H) & =\frac{1}{\pi^{2}} \int_{K \times L} \frac{\partial}{\partial \bar{z}} \tilde{f}(z) \frac{\partial}{\partial \bar{w}} \tilde{g}(w)(H-z)^{-1}(H-w)^{-1} d x d y d u d v \\
& =\frac{1}{\pi^{2}} \int_{K \times L} \frac{\partial}{\partial \bar{z}} \tilde{f}(z) \frac{\partial}{\partial \bar{w}} \tilde{g}(w) \frac{(H-w)^{-1}-(H-z)^{-1}}{w-z} d x d y d u d v .
\end{aligned}
$$

Then one uses the formula (easily proven using Stokes again)

$$
\frac{1}{\pi} \int_{K} \frac{\partial}{\partial \bar{z}} \tilde{f}(z) \frac{d x d y}{w-z}=\tilde{f}(w)
$$

the equivalent one for $\tilde{g}$ and one gets, changing variables to $z$, 


$$
\begin{aligned}
f(H) g(H) & =\frac{1}{\pi} \int_{K \cap L}\left(\tilde{g}(z) \frac{\partial}{\partial \bar{z}} \tilde{f}(z)+\tilde{f}(z) \frac{\partial}{\partial \bar{z}} \tilde{g}(z)\right)(H-z)^{-1} d x d y \\
& =\frac{1}{\pi} \int_{K \cap L} \frac{\partial}{\partial \bar{z}}(\tilde{f}(z) \tilde{g}(z))(H-z)^{-1} d x d y
\end{aligned}
$$

It remains to see that if $k(z)=\tilde{f}(z) \tilde{g}(z)-\widetilde{f g}(z)$, the integral of $\frac{\partial}{\partial \bar{z}} k(z)$ against the resolvent on $\mathbb{C}$ is zero. But this is again a consequence of Lemma 4.5, since $k$ has compact support and explicit computations yield $k(z)=O\left(y^{2}\right)$ as $y \rightarrow 0$ with $x$ fixed. The generalization to functions of $\mathcal{A}$ is proven along the same lines as Lemma 4.4 with Lemma 4.3.

c) The first point follows from $(H-z)^{-1^{*}}=(H-\bar{z})^{-1}$, the convergence in norm of (16) and the fact that $\widetilde{\bar{f}}(z)=\overline{\widetilde{f}(\bar{z})}$ if $\tau$ is even, which we can always assume. For the second point, take $f \in \mathcal{A}$ and $c>0$ such that $\|f\|_{\infty} \leq c$. Defining $g(x)=c-\left(c^{2}-|f(x)|^{2}\right)^{1 / 2}$, one checks that $g \in \mathcal{A}$ as well. The identity $f \bar{f}-2 c g+g^{2}=0$ in the algebra $\mathcal{A}$ implies with the above

$$
\begin{aligned}
& f(H) f(H)^{*}-2 c g(H)+g(H) g(H)^{*}=0 \\
& \Leftrightarrow f(H)^{*} f(H)+(c-g(H))^{*}(c-g(H))=c^{2} .
\end{aligned}
$$

Thus, for any $\psi \in \mathcal{H}$, it follows

$$
\|f(H) \psi\|^{2} \leq\|f(H) \psi\|^{2}+\|(c-g(H)) \psi\|^{2} \leq c^{2}\|\psi\|^{2},
$$

where $c \geq\|f\|_{\infty}$ is arbitrary.

d) Let us take $n=1$ and assume $\varsigma w>0$. We further choose

$$
\sigma(x, y)=\tau(\lambda y /<x>)
$$

where $\lambda \geq 1$ will be chosen large enough so that $w$ does not belong to the support of $\sigma$ and then kept fixed in the rest of the argument. The sole effect of this manipulation is to change the support of $\tau$, but everything we have done so far remains true for $\lambda>1$ and fixed. Let us define, for $m>0$ large,

$$
\Omega_{m}=\{(x, y)|| x \mid<m \text { and }<x>/ m<|y|<2 m\} .
$$

Then, by definition and Stokes,

$$
\begin{aligned}
r_{w}(H) & =\lim _{m \rightarrow \infty} \frac{1}{\pi} \int_{\Omega_{m}} \frac{\partial}{\partial \bar{z}} \tilde{r}_{w}(z)(H-z)^{-1} d x d y \\
& =\lim _{m \rightarrow \infty} \frac{1}{2 \pi i} \int_{\partial \Omega_{m}} \tilde{r}_{w}(z)(H-z)^{-1} d z
\end{aligned}
$$

where, since $n=1$,

$$
\tilde{r}_{w}(z)=\left(r_{w}(x)+r_{w}^{\prime}(x) i y\right) \sigma(x, y) .
$$

At this point, we want to replace $\tilde{r}_{w}(z)$ by $r_{w}(z)$ in (19). Indeed, it can be shown using the above explicit formula that 


$$
\lim _{m \rightarrow \infty}\left|\int_{\partial \Omega_{m}}\left(r_{w}(z)-\tilde{r}_{w}(z)\right)(H-z)^{-1} d z\right| \mid=0,
$$

by support considerations and elementary estimates on the different pieces of $\partial \Omega_{m}$. Admitting this fact we have

$$
\begin{aligned}
r_{w}(H) & =\lim _{m \rightarrow \infty} \frac{1}{2 \pi i} \int_{\partial \Omega_{m}} r_{w}(z)(H-z)^{-1} d z \\
& =\left.\operatorname{res}\left(r_{w}(z)(H-z)^{-1}\right)\right|_{z=w}=(H-w)^{-1},
\end{aligned}
$$

due to the analyticity of the resolvent inside $\Omega_{m}$.

We can now state the first Spectral Theorem for the set $C_{\infty}(\mathbb{R})$ of continuous functions that vanish at infinity

$$
C_{\infty}(\mathbb{R})=\{f \in C(\mathbb{R}) \mid \forall \epsilon>0, \exists K \text { compact with }|f(x)|<\epsilon \text { if } x \notin K\} .
$$

Theorem 4.1. There exists a unique linear map $f \mapsto f(H)$ from $C_{\infty}$ to $\mathcal{L}(\mathcal{H})$ which is a functional calculus.

Proof. Replacing $C_{\infty}$ by $\mathcal{A}$ we have existence. Now, $C_{0}^{\infty} \subset \mathcal{A} \subset C_{\infty}$ and it is a classical fact that $\bar{C}_{0}^{\infty}\|\cdot\|_{\infty}=C_{\infty}$, [RS]. Hence $\mathcal{A}$ is dense in $C_{\infty}$ in the sup norm. As $\|f(H)\| \leq\|f\|_{\infty} \forall f \in \mathcal{A}$, a density argument yields an extension of the map to $C_{\infty}$ with convergence in norm. It is routine to check that all properties listed in Proposition 4.1 remain true for $f \in C_{\infty}$. The uniqueness property is shown as follows. If there exists another functional calculus, then, by hypothesis, it must agree with ours on the set of functions $R$

$$
R=\overline{\left\{\sum_{i=1}^{n} \lambda_{i} r_{w_{i}}, \text { where } \lambda_{i} \in \mathbb{C}, w_{i} \notin \mathbb{R}\right\} .}
$$

But, it is a classical result also that the set $R$ satisfies the hypothesis of the Stone-Weierstrass Theorem ${ }^{\mathrm{b}}$ and $R=C_{\infty}$, so that the two functional calculus must coincide everywhere.

We shall pursue in two directions. We first want to show that any self-adjoint operator can be represented as a multiplication operator on some $L^{2}$ space. Then we shall extend the functional calculus to bounded measurable functions.

\section{2 $\quad L^{2}$ Spectral Representation}

Let $(H, D)$ be self-adjoint on $\mathcal{H}$.

\footnotetext{
${ }^{\mathrm{b}}$ Let $X$ be locally compact and consider $C_{\infty}(X)$. If $B$ is a subalgebra of $C_{\infty}(X)$ that separates points and satisfies $f \in B \Rightarrow \bar{f} \in B$, then $B$ is dense in $C_{\infty}(X)$ for $\|\cdot\|_{\infty}$
} 
Definition 4.3. A closed linear subspace $L$ of $\mathcal{H}$ is said invariant under $H$ if $(H-z)^{-1} L \subseteq L$ for any $z \notin \mathbb{R}$.

Remark 4.3. It is an exercise to show that if $\varphi \in L \cap D$, then $H \varphi \in L$, as expected.

Also, for any $\lambda \in \mathbb{R}, \operatorname{Ker}(H-\lambda)$ is invariant. If is is positive, the dimension of this subspace is called the multiplicity of the eigenvalue $\lambda$.

Lemma 4.7. If $L$ is invariant under $H=H^{*}$, then $L^{\perp}$ is invariant also. Moreover, $f(H) L \subseteq L$, for all $f \in C_{\infty}(\mathbb{R})$.

Proof. The first point is straightforward and the second follows from the approximation of the integral representation (16) of $f(H)$ for $f \in \mathcal{A}$ by a norm convergent limit of Riemann sums and by a density argument for $f \in C_{\infty}(\mathbb{R})$.

Definition 4.4. For $(H, D)$ self-adjoint on $\mathcal{H}$, the cyclic subspace generated by the vector $v \in \mathcal{H}$ is the subspace

$$
L=\overline{\operatorname{span}\left\{(H-z)^{-1} v, \quad z \notin \mathbb{R}\right\}} .
$$

Remark 4.4. i) Cyclic subspaces are invariant under $H$, as easily checked.

ii) If the vector $v$ chosen to generate the cyclic subspace is an eigenvector, then, this subspace is $\mathbb{C} v$.

iii) If the cyclic subspace corresponding to some vector $v$ coincides with $\mathcal{H}$, we say that $v$ is a cyclic vector for $H$.

iv) In the finite dimensional case, the matrix $H$ has a cyclic vector $v$ iff the spectrum of $H$ is simple, i.e. all eigenvalues have multiplicity one.

These subspaces allow to structure the Hilbert space with respect to the action of $H$.

Lemma 4.8. For $(H, D)$ self-adjoint on $\mathcal{H}$, there exists a sequence of orthogonal cyclic subspaces $L_{n} \subset \mathcal{H}$ with cyclic vector $v_{n}$ such that $\mathcal{H}=\overline{\oplus_{n=1}^{N} L_{n}}$, with $N$ finite or not.

Proof. As $\mathcal{H}$ is assumed to be separable, there exists an orthonormal basis $\left\{f_{j}\right\}_{j \in \mathbb{N}}$ of $\mathcal{H}$. Let $L_{1}$ be the subspace corresponding to $f_{1}$. By induction, let us assume orthogonal cyclic subspaces $L_{1}, L_{2}, \cdots, L_{n}$ are given. Let $m(n)$ be the smallest integer such that $f_{m(n)} \notin L_{1} \oplus \cdots \oplus L_{n}$ and let $g_{m(n)}$ be the component of $f_{m(n)}$ orthogonal to that subspace. We let $L_{n+1}$ be the cyclic subspace generated by the vector $g_{m(n)}$. Then we have $L_{n+1} \perp L_{r}$, for all $r \leq n$ and $f_{m(n)} \in L_{1} \oplus \cdots \oplus L_{n} \oplus L_{n+1}$. Then either the induction continues indefinitely and $N=\infty$, or at some point, such a $m(n)$ does not exist and the sum is finite.

The above allows us consider each $\left.H\right|_{L_{n}}, n=1,2 \cdots, N$ separately. Note, however, that the decomposition is not canonical. 
Theorem 4.2. Let $(H, D)$ be self-adjoint on $\mathcal{H}$, separable. Let $S=\sigma(H) \subset$ $\mathbb{R}$. Then there exists a finite positive measure $\mu$ on $S \times \mathbb{N}$ and a unitary operator $U: \mathcal{H} \rightarrow L^{2} \equiv L^{2}(S \times \mathbb{N}, d \mu)$ such that if

$$
\begin{aligned}
h: & : S \times \mathbb{N} \rightarrow \mathbb{R} \\
& (x, n) \mapsto x
\end{aligned}, \quad \text { then } \quad \xi \in \mathcal{H} \text { belongs to } D \Longleftrightarrow h U \xi \in L^{2} .
$$

Moreover,

$$
\begin{aligned}
& U H U^{-1} \psi=h \psi, \forall \psi \in U(D) \subset L^{2}(S \times \mathbb{N}, d \mu) \text { and } \\
& U f(H) U^{-1} \psi=f(h) \psi, \quad \forall f \in C_{\infty}(\mathbb{R}), \quad \psi \in L^{2}(S \times \mathbb{N}, d \mu) .
\end{aligned}
$$

This Theorem will be a Corollary of the

Theorem 4.3. Let $(H, D)$ be self-adjoint on $\mathcal{H}$ and $S=\sigma(H) \subset \mathbb{R}$. Further assume that $H$ admits a cyclic vector $v$. Then, there exists a finite positive measure $\mu$ on $S$ and a unitary operator $U: \mathcal{H} \rightarrow L^{2}(S, d \mu) \equiv L^{2}$ such that if

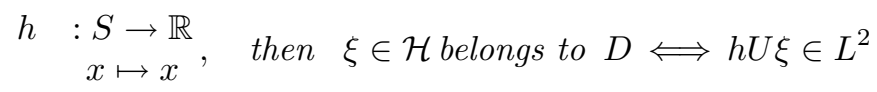

and

$$
U H U^{-1} \psi=h \psi \forall \psi \in U(D) \subset L^{2}(S, d \mu) .
$$

Proof. (of Theorem 4.3). Let the linear functional $\Phi: C_{\infty}(\mathbb{R}) \rightarrow \mathbb{C}$ be defined by

$$
\Phi(f)=\langle v \mid f(H) v\rangle .
$$

The functional calculus shows that $\Phi(\bar{f})=\overline{\Phi(f)}$. And if $0 \leq f \in C_{\infty}(\mathbb{R})$, then, with $g=\sqrt{f}$, we have

$$
\Phi(f)=\|g(H) v\|^{2} \geq 0 \text {, i.e. } \Phi \text { is positive. }
$$

Thus, by Riesz-Markov Theorem ${ }^{\mathrm{c}}$, there exists a positive measure on $\mathbb{R}$ such that

$$
\langle v \mid f(H) v\rangle=\int_{\mathbb{R}} f(x) d \mu(x), \forall f \in C_{\infty}(\mathbb{R}) .
$$

Since in case supp $(f) \cap \sigma(H)=\emptyset, f(H)$ is zero, we deduce that supp $(\mu) \subseteq$ $S=\sigma(H)$. Also, note that $f$ above belongs to $L^{2}(S, d \mu)$, since

$$
\int|f(x)|^{2} d \mu(x)=\left\langle v \mid f(H)^{*} f(H) v\right\rangle \leq\left\|f^{2}\right\|_{\infty}\|v\|^{2}<\infty .
$$

Consider the linear map $T: C_{\infty}(\mathbb{R}) \rightarrow L^{2}$ such that $T f=f$. It satisfies for any $f, g \in C_{\infty}(\mathbb{R})$

${ }^{\mathrm{c}}$ If $X$ is a locally compact space, any positive linear functional $l$ on $C_{\infty}(X)$ is of the form $l(f)=\int_{X} f d \mu$, where $\mu$ is a (regular) Borel measure with finite total mass. 


$$
\begin{aligned}
\langle T g \mid T f\rangle_{L^{2}} & =\int_{S} \bar{g}(x) f(x) d \mu(x)=\Phi(\bar{g} f) \\
& =\left\langle v \mid g^{*}(H) f(H) v\right\rangle_{\mathcal{H}}=\langle g(H) v \mid f(H) v\rangle_{\mathcal{H}} .
\end{aligned}
$$

Defining

$$
M=\left\{f(H) v \in \mathcal{H} \mid f \in C_{\infty}(\mathbb{R})\right\},
$$

we have existence of an onto isomorphism $U$ such that

$$
U: M \rightarrow C_{\infty}(\mathbb{R}) \subseteq L^{2} \text { such that } U f(H) v=f .
$$

Now, $M$ is dense in $\mathcal{H}$ since $v$ is cyclic and $C_{\infty}(\mathbb{R})$ is dense in $L^{2}$, so that $U$ admits a unitary extension from $\mathcal{H}$ to $L^{2}$.

Let $f_{1}, f_{2}, f \in C_{\infty}(\mathbb{R})$ and $\psi_{i}=f_{i}(H) v \in \mathcal{H}$. Then

$$
\begin{aligned}
\left\langle\psi_{2} \mid f(H) \psi_{1}\right\rangle_{\mathcal{H}} & =\left\langle f_{2}(H) v \mid\left(f f_{1}\right)(H) v\right\rangle_{\mathcal{H}} \\
& =\int_{S} \bar{f}_{2}(x) f(x) f_{1}(x) d \mu(x)=\left\langle U \psi_{2} \mid f U \psi_{1}\right\rangle_{L^{2}},
\end{aligned}
$$

where $f$ denotes the obvious multiplication operator. In particular, if $f(x)=$ $r_{w}(x)=(x-w)^{-1}$, we deduce that for all $\xi \in L^{2}$ and all $w \notin \mathbb{R}$

$$
U r_{w}(H) U^{-1} \xi=U(H-w)^{-1} U^{-1} \xi=r_{w} \xi .
$$

Thus, $U$ maps $\operatorname{Ran}(H-w)$ to $\operatorname{Ran}\left(r_{w}\right)$, i.e. $D$ and $\left\{\xi \in L^{2} \mid x \xi(x) \in L^{2}\right\}$ are in one to one correspondence.

If $\xi \in L^{2}$ and $\psi=r_{w} \xi$, then $\psi \in D(h)$, where $D(h)$ is the domain of the multiplication operator by $h: x \mapsto x$. Then, with (20)

$U H U^{-1} \psi=U H U^{-1} r_{w} \xi=U H r_{w}(H) U^{-1} \xi=w r_{w} \xi+\xi=x r_{w} \xi=h \psi$.

Proof. (of Theorem 4.2). We know $\mathcal{H}=\overline{\oplus_{n} L_{n}}$ with cyclic subspaces $L_{n}$ with vectors $v_{n}$. We will assume $\left\|v_{n}\right\|=1 / 2^{n}, \forall n \in \mathbb{N}$. By Theorem 4.2, there exist $\mu_{n}$ of mass $\int_{S} d \mu_{n}=\left\|v_{n}\right\|^{2}=2^{-2 n}$ and unitary operators $U_{n}: L_{n} \rightarrow L^{2}\left(S, d \mu_{n}\right)$ such that $H_{n}=\left.H\right|_{L_{n}}$ is unitarily equivalent to the multiplication by $x$ on $L^{2}\left(S, d \mu_{n}\right)$. Defining $\mu$ on $S \times \mathbb{N}$ by imposing $\left.\mu\right|_{S \times\{n\}}=\mu_{n}$ and $U$ by $\oplus_{n} U_{n}$, we get the result.

In case $\mathcal{H}=\mathbb{C}^{n}$ and $H=H^{*}$ has simple eigenvalues $\lambda_{j}$ with associated eigenvectors $\psi_{j}$, the measure can be chosen as $\mu=\sum_{j} \delta\left(x-\lambda_{j}\right)$ and $L^{2}=L^{2}(\mathbb{R}, d \mu)=\mathbb{C}^{n}$. Note also that $\tilde{\mu}=\sum_{j} a_{j} \delta\left(x-\lambda_{j}\right)$, where $a_{j}>0$ is as good a measure as $\mu$ to represent $\mathbb{C}^{n}$ as $L^{2}(\mathbb{R}, \tilde{d \mu})$.

Let us now extend our Spectral Theorem to $\mathcal{B}(\mathbb{R})$, the set of bounded Borel functions on $\mathbb{R}$. 
Definition 4.5. We say that $f_{n} \in \mathcal{B}(\mathbb{R})$ is monotonically increasing to $f \in \mathcal{B}(\mathbb{R})$, if $f_{n}(x)$ increases monotonically to $f(x)$ for any $x \in \mathbb{R}$.

Thus, $\left\|f_{n}\right\|=\sup _{x \in \mathbb{R}}\left|f_{n}(x)\right|$ is uniformly bounded in $n$.

Theorem 4.4. There exists a unique functional calculus $f \rightarrow f(H)$ from $\mathcal{B}(\mathbb{R})$ to $\mathcal{L}(\mathcal{H})$ if one imposes $s-\lim _{n \rightarrow \infty} f_{n}(H)=f(H)$ if $f_{n} \in \mathcal{B}(\mathbb{R})$ converges monotonically to $f \in \mathcal{B}(\mathbb{R})$.

Recall that s-lim means limit in the strong sense, i.e. s-lim $A_{n}=A$ in $\mathcal{L}(\mathcal{H})$ is equivalent to $\lim _{n} A_{n} \varphi=A \varphi$, in $\mathcal{H}, \forall \varphi \in \mathcal{H}$.

Proof. Consider existence first. By unitary equivalence, we identify $\mathcal{H}$ and $L^{2}(S \times \mathbb{N}, d \mu)$ and $H$ by multiplication by $h:(x, n) \mapsto x$. We define $f(H)$ for $f \in \mathcal{B}(\mathbb{R})$ by

$$
f(H) \psi(x, n)=f(h(x, n)) \psi(x, n) \text { on } L^{2}(S \times \mathbb{N}, d \mu),
$$

which is easily shown to satisfy the properties of a functional calculus. Then, by the dominated convergence Theorem, if $f_{n}$ converges monotonically to $f$ :

$$
\lim _{n \rightarrow \infty} f_{n}(H) \psi(x, m)=f(H) \psi(x, m) .
$$

Uniqueness is shown as follows. Consider two functional calculus with the mentioned properties. Let $\mathcal{C}$ be the subset of $\mathcal{B}(\mathbb{R})$ on which they coincide. We know $C_{\infty}(\mathbb{R}) \subset \mathcal{C}$ and $\mathcal{C}$ is closed by taking monotone limits. But the smallest set of functions containing $C_{\infty}(\mathbb{R})$ which is closed under monotone limits is $\mathcal{B}(\mathbb{R})$, see $[\mathrm{RS}]$.

Remark 4.5. It all works the same if one considers functions $f_{n}$ that converge pointwise to $f$ and $\operatorname{such}_{\text {that }} \sup _{n}\left\|f_{n}\right\|_{\infty}<\infty$

We have the following Corollary concerning the resolvent.

Corollary 4.1. With the hypotheses and notations above, $\sigma(H)$ is the essential range of $h$ in $L^{2}(S, d \mu)$ and

$$
\left\|(H-z)^{-1}\right\|=\frac{1}{\operatorname{dist}(z, \sigma(H))}
$$

Proof. This follows from Theorem 4.3 and our study of multiplication operators.

Another instance where our previous study of multiplication operators is useful is the case of constant coefficient differential operators on $\mathcal{S}\left(\mathbb{R}^{N}\right)$, the set of Schwartz functions. Such an operator $L$ is defined by a finite sum of the form 


$$
L=\sum_{\alpha} a_{\alpha} D^{\alpha}
$$

where

$$
\alpha=\left(\alpha_{1}, \cdots, \alpha_{N}\right) \in \mathbb{N}^{N}, \quad a_{\alpha} \in \mathbb{C}, \quad D^{\alpha_{j}}=\frac{\partial^{\alpha_{j}}}{\partial x_{j}^{\alpha_{j}}}, D^{\alpha}=D^{\alpha_{1}} \cdots D^{\alpha_{N}},
$$

and $L$ acts on functions in $\mathcal{S}\left(\mathbb{R}^{N}\right) \subset L^{2}\left(\mathbb{R}^{N}\right)$. This set of functions being invariant under Fourier transformation $\mathcal{F}$, this operator is unitarily equivalent to

$$
\mathcal{F} L \mathcal{F}^{-1}=\sum_{\alpha} a_{\alpha}(i k)^{\alpha}, \text { on } \mathcal{S}\left(\mathbb{R}^{N}\right) \subset L^{2}\left(\mathbb{R}^{N}\right) .
$$

The function $\sum_{\alpha} a_{\alpha}(i k)^{\alpha}$ is called the symbol of the differential operator. It is not difficult to get the following

Proposition 4.2. Let $L$ be the differential operator on $\mathbb{R}^{n}$ with constant coefficients defined in (21). Then $L$ is symmetric iff its symbol is real valued in $\mathbb{R}^{N}$. In that case, $\bar{L}$ is self-adjoint and

$$
\sigma(\bar{L})=\overline{\left\{\sum_{\alpha} a_{\alpha}(i k)^{\alpha} \mid k \in \mathbb{R}^{N}\right\}}
$$

Let us finally introduce spectral projectors in the general case of unbounded operators.

Theorem 4.5. Let $(H, D)$ be self-adjoint on $\mathcal{H}$ and $(a, b)$ an open interval. Let $f_{n}$ be an increasing sequence of non-negative continuous functions on $\mathbb{R}$ with support in $(a, b)$ that converges to $\chi_{(a, b)}$, the characteristic function of $(a, b)$. Then

$$
s-\lim _{n} f_{n}(H)=P_{(a, b)},
$$

a canonical orthogonal projector, independent of $\left\{f_{n}\right\}$, that satisfies

$$
P_{(a, b)} H \subset H P_{(a, b)} \text {, and } P_{(a, b)}=0 \Longleftrightarrow(a, b) \cap \sigma(H)=\emptyset
$$

Proof. The existence of the limit is ensured by Theorem 4.4 and and its properties are immediate.

Remark 4.6. The fact that $P_{(a, b)}$ is a projector follows from the identity $\chi_{(a, b)}=\chi_{(a, b)}^{2}$, which makes $\chi_{(a, b)}$ a projector, when viewed as a multiplication operator.

These projectors are called spectral projectors and their range $L_{(a, b)}=P_{(a, b)} \mathcal{H}$ are called spectral subspaces. These spectral subspaces satisfy

$$
L_{(a, b)} \simeq L^{2}\left(E_{(a, b)}, d \mu\right), \text { where } E_{(a, b)}=\{(x, n) \mid a<h(x, n)<b\},
$$


and $\simeq$ denotes the unitary equivalence constructed in Theorem 4.2

It is also customary to represent a self-adjoint operator $H$ by the Stieltjes integral

$$
H=\int_{-\infty}^{\infty} \lambda d E(\lambda)
$$

where $E(\lambda)=P(-\infty, \lambda)$ is projection operator valued. Let us justify this in case $H$ has a cyclic vector, the general case following immediately. By polarization, it is enough to check that for $\xi \in D$

$$
\langle\xi \mid H \xi\rangle_{\mathcal{H}}=\int \lambda d\langle\xi \mid E(\lambda) \xi\rangle=\int \lambda d\|E(\lambda) \xi\|^{2} .
$$

By unitary equivalence to $L^{2}(\mathbb{R}, d \mu)$, if $\psi=U \xi$

$$
\begin{aligned}
d\|E(\lambda) \xi\|^{2} & =d \int_{-\infty}^{\infty}\left|\chi_{(-\infty, \lambda)}(x) \psi(x)\right|^{2} d \mu(x) \\
& =d \int_{-\infty}^{\lambda}|\psi(x)|^{2} d \mu(x)=|\psi(\lambda)|^{2} d \mu(\lambda) .
\end{aligned}
$$

Hence

$$
\langle\xi \mid H \xi\rangle_{\mathcal{H}}=\int \lambda|\psi(\lambda)|^{2} d \mu(\lambda)=\langle\psi \mid h \psi\rangle_{L^{2}}
$$

We close this Section about the Spectral Theorem by some results in perturbation theory of unbounded operators.

Definition 4.6. Let $(H, D)$ and $\left(H_{n}, D_{n}\right)$ be a sequence of self-adjoint operators on $\mathcal{H}$. We say that $H_{n} \rightarrow H$ in the norm resolvent sense if

$$
\lim _{n \rightarrow \infty}\left\|\left(H_{n}+i\right)^{-1}-(H+i)^{-1}\right\|=0 .
$$

The point $i \in \mathbb{C}$ plays no particular role as the following Lemma shows.

Lemma 4.9. If $z=x+i y \in \mathbb{C} \backslash \mathbb{R}$, and $g(x, y)=\sup _{h \in \mathbb{R}}|(h+i) /(h-x-i y)|$ then, there exists a constant $c$ such that

$$
\left\|\left(H_{n}-z\right)^{-1}-(H-z)^{-1}\right\| \leq c g(x, y)\left\|\left(H_{n}+i\right)^{-1}-(H+i)^{-1}\right\| .
$$

Proof. Once the identity

$$
\begin{aligned}
& \left(H_{n}-z\right)^{-1}-(H-z)^{-1}= \\
& \quad\left(H_{n}+i\right)\left(H_{n}-z\right)^{-1}\left(\left(H_{n}+i\right)^{-1}-(H+i)^{-1}\right)(H+i)(H-z)^{-1}
\end{aligned}
$$

is established, the Lemma is a consequence of the bound following from the Spectral Theorem

$$
\left\|(H+i)(H-z)^{-1}\right\| \leq g(x, y) .
$$

If one doesn't take care of domain issues, (22) is straightforward. We refer to [D] for a careful proof of (22). 
Remark 4.7. If $z \in \rho(H) \cap \rho\left(H_{n}\right)$, the result is similar.

Theorem 4.6. If $H_{n} \rightarrow H$ in the norm resolvent sense, then

$$
\lim _{n \rightarrow \infty}\left\|f\left(H_{n}\right)-f(H)\right\|=0, \forall f \in C_{\infty} .
$$

Remark 4.8. The result is not generally true if $f \in \mathcal{B}(\mathbb{R})$. Consider spectral projectors, for instance. See also Corollary 4.2 below.

Proof. If $f \in \mathcal{A}$, the definition yields,

$$
\begin{aligned}
\left\|f(H)-f\left(H_{n}\right)\right\| & \leq \frac{1}{\pi} \int_{\mathbb{C}}\left|\frac{\partial \tilde{f}(z)}{\partial \bar{z}}\right|\left\|\left(H_{n}-z\right)^{-1}-(H-z)^{-1}\right\| d x d y \\
& \leq \frac{4 c}{\pi} \int_{\mathbb{C}}\left|\frac{\partial \tilde{f}(z)}{\partial \bar{z}}\right| g(x, y) d x d y\left\|\left(H_{n}+i\right)^{-1}-(H+i)^{-1}\right\| .
\end{aligned}
$$

It is not difficult to see that the last integral is finite, due to the properties of $\frac{\partial \tilde{f}(z)}{\partial \bar{z}}$ and of $g(x, y)$. The convergence in norm is established for $f \in \mathcal{A}$ and the extension of the result to $f \in C_{\infty}(\mathbb{R})$ comes from the extension of the functional calculus to those $f$ 's and by density.

We have the following spectral consequences.

Corollary 4.2. If $H_{n} \rightarrow H$ in the norm resolvent sense, we have convergence of the spectrum of $H_{n}$ to that of $H$ in the following sense:

$$
\begin{aligned}
& \lambda \in \mathbb{R} \backslash \sigma(H) \Rightarrow \lambda \notin \sigma\left(H_{n}\right), \text { n large enough } \\
& \lambda \in \sigma(H) \Rightarrow \exists \lambda_{n} \in \sigma\left(H_{n}\right), \text { such that } \lim _{n \rightarrow \infty} \lambda_{n}=\lambda .
\end{aligned}
$$

Proof. If $\lambda \in \mathbb{R} \backslash \sigma(H)$, there exists $f \in C_{0}^{\infty}(\mathbb{R})$ whose support is disjoint from $\sigma(H)$ and which is equal to 1 in a neighborhood of $\lambda$. Then, Theorem 4.6 implies $\left\|f\left(H_{n}\right)\right\| \rightarrow 0$ and, in turn, the Spectral Theorem implies $\lambda \notin \sigma\left(H_{n}\right)$ if $\left\|f\left(H_{n}\right)\right\|<1$. Conversely, if $\lambda \in \sigma(H)$, pick an $\epsilon>0$ and a $f \in C_{0}^{\infty}(\mathbb{R})$ such that $f(\lambda)=1$ and $\operatorname{supp}(f) \subset(\lambda-\epsilon, \lambda+\epsilon)$. From $\lim _{n}\left\|f\left(H_{n}\right)\right\|=1$ follows that $\sigma\left(H_{n}\right) \cap(\lambda-\epsilon, \lambda+\epsilon) \neq \emptyset$, if $n$ is as large as we wish.

\section{Stone's Theorem, Mean Ergodic Theorem and Trotter Formula}

The Spectral Theorem allows to prove easily Stone's Theorem, which characterizes one parameter evolution groups which we define below. Such groups are those giving the time evolution of a wave function $\psi$ in Quantum Mechanics governed by the Schrödinger equation

$$
i \hbar \frac{\partial}{\partial t} \psi(t)=H \psi(t), \quad \text { with } \psi(0)=\psi_{0},
$$

where $H$ is the Hamiltonian. 
Definition 5.1. A one-parameter evolution group on a Hilbert space is a family $\{U(t)\}_{t \in \mathbb{R}}$ of unitary operators satisfying $U(t+s)=U(t) U(s)$ for all $t, s \in \mathbb{R}$ and $U(t)$ is strongly continuous in $t$ on $\mathbb{R}$.

Remark 5.1. It is easy to check that strong continuity at 0 is equivalent to strong continuity everywhere and that weak continuity is equivalent to strong continuity in that setting.

Actually, we have equivalence between the following statements: the map $t \mapsto\langle\varphi \mid U(t) \psi\rangle$ is measurable for all $\varphi, \psi$ and $U(t)$ is strongly continuous, see [RS] for a proof.

Theorem 5.1. Let $(A, D)$ be self-adjoint on $\mathcal{H}$ and $U(t)=e^{i t A}$ given by functional calculus. Then

a) $\{U(t)\}_{t \in \mathbb{R}}$ forms a one parameter evolution group and $U(t): D \rightarrow D$ for $t \in \mathbb{R}$.

b) For any $\psi \in D$,

$$
\frac{U(t) \psi-\psi}{t} \rightarrow i A \psi \text { as } t \rightarrow 0
$$

c) Conversely,

$$
\lim _{t \rightarrow 0} \frac{U(t) \psi-\psi}{t} \text { exists } \Rightarrow \psi \in D .
$$

Proof. a) follows from the Functional Calculus and the properties of $x \mapsto$ $f_{t}(x)=e^{i t x}$. Similarly b) is a consequence of the functional calculus (see Theorem 4.4) applied to $x \mapsto g_{t}(x)=\left(e^{i t x}-1\right) / t$ and of the estimate $\mid e^{i x}-$ $1|\leq| x \mid$.

c) Define

$D(B)=\left\{\psi \mid \lim _{t \rightarrow 0} \frac{U(t) \psi-\psi}{t}\right.$ exists $\}$, and $B \psi=\lim _{t \rightarrow 0} \frac{U(t) \psi-\psi}{i t}$ on $D(B)$.

One checks that $B$ is symmetric and b) implies $B \supseteq A$. But $A \subseteq B \subseteq \bar{B}$ and $A=A^{*}$ is closed, thus $A=B$.

Remark 5.2. The formula (23) defines the so-called infinitesimal generator of the evolution group $U(t)$.

The converse of that result is Stone's Theorem.

Theorem 5.2. If $\{U(t)\}_{t \in \mathbb{R}}$ forms a one parameter evolution group on $\mathcal{H}$, then there exists $(A, D)$ self-adjoint on $\mathcal{H}$ such that $U(t)=e^{i A t}$.

Proof. The idea of the proof is to define $A$ as the infinitesimal generator on a set of good vectors and show that $A$ is essentially self-adjoint. Then one shows that $U(t)={ }^{i \bar{A} t}$.

Let $f \in C_{0}^{\infty}(\mathbb{R})$ and define 


$$
\varphi_{f}=\int_{\mathbb{R}} f(t) U(t) \varphi d t, \forall \varphi \in \mathcal{H} .
$$

Let $D$ be the set of finite linear combinations of such $\varphi_{f}$, with different $\varphi$ and $f$.

1) $D$ is dense: Let $j_{\epsilon}(x)=j(x / \epsilon) / \epsilon$, where $0 \leq j \in C_{0}^{\infty}$ with support in $[-1,1]$ and $\int j(x) d x=1$. Then, for any $\varphi$,

$$
\begin{aligned}
\left\|\varphi_{j_{\epsilon}}-\varphi\right\| & =\left\|\int j_{\epsilon}(t)(U(t) \varphi-\varphi) d t\right\| \\
& \leq\left(\int_{-\infty}^{\infty} j_{\epsilon}(t) d t\right) \sup _{|t| \leq \epsilon}\|U(t) \varphi-\varphi\| \rightarrow 0 \text { as } \epsilon \rightarrow 0 .
\end{aligned}
$$

2) Infinitesimal generator on $D$ : Let $\varphi_{f} \in D$.

$$
\begin{aligned}
\frac{(U(s)-I)}{s} \varphi_{f}= & \int_{\mathbb{R}} f(t) \frac{U(t+s)-U(t)}{s} \varphi d t \\
= & \int_{\mathbb{R}} \frac{f(\tau-s)-f(\tau)}{s} U(\tau) \varphi d \tau \\
& s \rightarrow 0 \\
& \longrightarrow-\int_{\mathbb{R}} f^{\prime}(\tau) U(\tau) \varphi d \tau=\varphi_{-f^{\prime}}
\end{aligned}
$$

Hence, we set for $\varphi_{f} \in D$,

$$
A \varphi_{f}=\frac{1}{i} \varphi_{-f^{\prime}}=\lim _{t \rightarrow 0} \frac{U(t)-I I}{i t} \varphi_{f}
$$

and it is easily checked that

$$
U(t): D \rightarrow D, \quad A: D \rightarrow D, \quad \text { and } \quad U(t) A \varphi_{f}=A U(t) \varphi_{f}=\frac{1}{i} \varphi_{-f^{\prime}(\cdot-t)} .
$$

Moreover, for $\varphi_{f}, \varphi_{g} \in D$,

$$
\left\langle\varphi_{g} \mid A \varphi_{f}\right\rangle=\lim _{s \rightarrow 0}\left\langle\varphi_{f} \mid \frac{U(s)-I I}{i s} \varphi_{g}\right\rangle=\lim _{s \rightarrow 0}\left\langle\frac{U(-s)-I I}{-i s} \varphi_{f} \mid \varphi_{g}\right\rangle=\left\langle A \varphi_{f} \mid \varphi_{g}\right\rangle,
$$

so that $A$ is symmetric.

3) $A$ is essentially self-adjoint: Assume there exists $\psi \in D^{*}=D\left(A^{*}\right)$ such that $A^{*} \psi=i \psi$. Then, $\forall \varphi \in D$,

$$
\frac{d}{d t}\langle\psi \mid U(t) \varphi\rangle=\langle\psi \mid i A U(t) \varphi\rangle=i\left\langle A^{*} \psi \mid U(t) \varphi\right\rangle=\langle\psi \mid U(t) \varphi\rangle .
$$

Hence, solving the differential equation,

$$
\langle\psi \mid U(t) \varphi\rangle=\langle\psi \mid \varphi\rangle e^{t}
$$


As $\|U(t)\|=1$, this implies $\langle\psi \mid \varphi\rangle=0$, so that $\psi=0$ as $D$ is dense. A similar reasoning holds for any $\chi \in \operatorname{Ker}\left(A^{*}+i\right)$, so that $A$ is essentially self-adjoint and $\bar{A}$ is self-adjoint.

4) $U(t)=e^{i \bar{A} t}$ : Let $\varphi \in D \subseteq \bar{D}=D(\bar{A})$. On the one hand,

$$
e^{i \bar{A} t} \varphi \in \bar{D} \quad \text { and } \quad \frac{d}{d t} e^{i \bar{A} t} \varphi=i \bar{A} e^{i \bar{A} t} \varphi
$$

by b) Theorem 5.1. On the other hand, $U(t) \varphi \in D \subset \bar{D}$ for all $t$. Thus, introducing $\psi(t)=U(t) \varphi-e^{i \bar{A} t} \varphi$; we compute

$$
\psi^{\prime}(t)=i A U(t) \varphi-i \bar{A} e^{i \bar{A} t} \varphi=i \bar{A} \psi(t), \quad \text { with } \quad \psi(0)=0,
$$

so that $\frac{d}{d t}\|\psi(t)\|^{2} \equiv 0$, hence $\psi(t) \equiv 0$. As $D$ is dense, $e^{i \bar{A} t} \equiv U(t)$.

Examining the above proof, one deduces the following Corollary which can be useful in applications.

Corollary 5.1. Let $(A, D)$ be self-adjoint on $\mathcal{H}$ et $E \subset D$ be dense. If, for all $t \in \mathbb{R}, e^{i t A}: E \rightarrow E$, then $\left(\left.A\right|_{E}, E\right)$ is essentially self-adjoint.

Remark 5.3. i) In the situation of the Corollary, one says that $E$ is a core for A.

ii) The solution to the following equation, in the strong sense,

$$
\frac{d}{d t} \varphi(t)=i A \varphi(t), \quad \forall t \in \mathbb{R} \varphi(0)=\varphi_{0} \in D
$$

is unique and is given by $\varphi(t)=e^{i A t} \varphi_{0}$.

In case $A$ is bounded, the evolution group generated by $A$ can be obtained from the power series of the exponential. This relation remains true in a certain sense when $A$ is unbounded and self-adjoint. Indeed, if $\varphi$ belongs to the dense set $\cup_{M \geq 0} P(-M, M) \mathcal{H}$, where $P(-M, M)$ denotes the spectral projectors of $A$, we get

$$
\sum_{k=0}^{N} \frac{(i t A)^{k}}{k !} \varphi \rightarrow e^{i t A} \varphi, \quad \text { as } \quad N \rightarrow \infty .
$$

This formula makes sense due to the fact that $\varphi \in \cap_{n \geq 0} D\left(A^{n}\right)$, where $D\left(A^{n}\right)$ is the domain of $A^{n}$. Stone's Theorem provides a link between evolution groups and self-adjoint generators, therefore one can expect a relation between essentially self-adjoint operators and the existence of sufficiently many vectors for which the above formula makes sense. 
Definition 5.2. Let $A$ be an operator on a Hilbert space $\mathcal{H}$. A vector $\varphi \in$ $\cap_{n \geq 0} D\left(A^{n}\right)$ is called an analytic vector if

$$
\sum_{k=0}^{\infty} \frac{\left\|A^{k} \varphi\right\|}{k !} t^{k}<\infty, \quad \text { for some } t>0 .
$$

The relation alluded to above is provided by the following criterion for essential self-adjointness.

Theorem 5.3 (Nelson's Analytic Vector Theorem). Let $(A, D)$ be symmetric on a Hilbert space $\mathcal{H}$. If $D$ contains a total set of analytic vectors, then $(A, D)$ is essentially self-adjoint.

We refer the reader to [RS] for a proof and we proceed by providing a link between the discrete spectrum of the self-adjoint operator $H$ with the evolution operator $e^{i t H}$ it generates. This is the so-called

Theorem 5.4 (Mean Ergodic Theorem). Let $P_{\lambda}$ be the spectral projector on an eigenvalue $\lambda$ of a self-adjoint operator $H$ of domain $D \in \mathcal{H}$. Then

$$
P_{\lambda}=s-\lim _{t_{2}-t_{1} \rightarrow \infty} \frac{1}{t_{2}-t_{1}} \int_{t_{1}}^{t_{2}} e^{i t H} e^{-i t \lambda} d t .
$$

Proof. One can assume $\lambda=0$ by considering $H-\lambda$ if necessary.

i) If $\varphi \in P_{0} \mathcal{H}$, then $H \varphi=0$ and $e^{i t H} \varphi=\varphi$, so that

$$
\frac{1}{t_{2}-t_{1}} \int_{t_{1}}^{t_{2}} e^{i t H} \varphi d t=\varphi=P_{0} \varphi, \text { for any } t_{1}, t_{2} .
$$

ii) If $\varphi \in \operatorname{Ran}(H)$, i.e. $\varphi=H \psi$ for some $\psi \in D$, then

$$
e^{i t H} \varphi=e^{i t H} H \psi=-i \frac{d}{d t} e^{i t H} \psi,
$$

so that we can write

$$
\frac{1}{t_{2}-t_{1}} \int_{t_{1}}^{t_{2}} e^{i t H} \varphi d t=-\frac{i}{t_{2}-t_{1}}\left(e^{i t_{2} H}-e^{i t_{1} H}\right) \psi \rightarrow 0=P_{0} \psi .
$$

The result is thus proven for $\operatorname{Ran}(H)$ and $\operatorname{Ran}\left(P_{0}\right)$. As the integral is uniformly bounded, the result is true on $\overline{\operatorname{Ran}(H)}$ as well and we conclude by

$$
\mathcal{H}=\overline{\operatorname{Ran}(H)} \oplus \operatorname{Ker}(H)=\overline{\operatorname{Ran}(H)} \oplus P_{0} \mathcal{H},
$$

which follows from $H=H^{*}$.

With a little more efforts, one can prove in the same vein 
Theorem 5.5 (Von Neumann's Mean Ergodic Theorem). If $V$ is such that $\left\|V^{n}\right\| \leq C$, uniformly in $n$ and $P_{1}$ projects on $\operatorname{Ker}(V-I I)$, then

$$
\frac{1}{N} \sum_{n=0}^{N-1} V^{n} \varphi \rightarrow P_{1} \varphi, \text { as } N \rightarrow \infty .
$$

Remark 5.4.0) The projector $P_{1}$ is not necessarily self-adjoint.

i) The projection on $\operatorname{Ker}(V-\lambda)$ where $|\lambda|=1$ is obtained by replacing $V$ by $V / \lambda$ in the Theorem.

ii) It follows from the assumption that $\sigma(V) \subseteq\{z|| z \mid \leq 1\}$, since the spectral radius $\operatorname{spr}(V)=\lim _{n \rightarrow \infty}\left\|V^{n}\right\|^{1 / n}=1$.

iii) A proof can be found in $[\mathrm{Y}]$.

Another link between the spectrum of its generator and the behaviour of an evolution group arises when a vector is transported away from its initial value at $t=0$ by the evolution exponentially fast as $|t| \rightarrow \infty$.

Proposition 5.1. Let $(H, D)$ be self-adjoint on $\mathcal{H}$ and assume there exists a normalized vector $\varphi \in \mathcal{H}$ such that, for any $t \in \mathbb{R}$ and for some positive constants $A, B$,

$$
\left|\left\langle\varphi \mid e^{i t H} \varphi\right\rangle\right| \leq A e^{-B|t|} .
$$

Then $\sigma(H)=\mathbb{R}$.

Proof. Taking $\varphi$ as first vector in the decomposition provided in Lemma 4.8, we have by the Spectral Theorem, that on $L^{2}\left(d \mu_{1}\right)$, the restriction of $L^{2}(d \mu)$ unitary equivalent to that first cyclic subspace,

$$
\varphi \simeq 1, \quad e^{i t H} \varphi \simeq e^{i t x} 1,
$$

so that

$$
\left\langle\varphi \mid e^{i t H} \varphi\right\rangle=\int e^{i t x} d \mu_{1}(x) \equiv f(t) .
$$

This $f$ admits a Fourier transform $\omega \mapsto \hat{f}(\omega)$ which is analytic in a strip $\{\omega|| \Im \omega \mid<B\}$. Therefore, we have $d \mu_{1}(x)=\hat{f}(x) d x$ and the support of $d \mu_{1}=\mathbb{R}$. Hence, $\sigma(H) \supset \mathbb{R}$.

Let us close this Section by a result concerning evolution groups generated by sums of self-adjoint operators.

Theorem 5.6 (Trotter product formula). Let $\left(A, D_{A}\right),\left(B, D_{B}\right)$ be selfadjoint and $A+B$ be essentially self-adjoint on $D_{A} \cap D_{B}$. Then

$$
e^{i(A+B) t}=s-\lim _{n \rightarrow \infty}\left(e^{i t A / n} e^{i t B / n}\right)^{n}, \quad \forall t \in \mathbb{R} .
$$

If, moreover, $A$ and $B$ are bounded from below,

$$
e^{-(A+B) t}=s-\lim _{n \rightarrow \infty}\left(e^{-t A / n} e^{-t B / n}\right)^{n}, \quad \forall t \geq 0 .
$$


Remark 5.5. The operator $e^{-C t}, t \geq 0$ for $C$ self-adjoint and bounded below can be defined via the Spectral Theorem applied to the function $f$ defined as follows: $f(x)=e^{-x}$, if $x \geq x_{0}$ and $f(x)=0$ otherwise, with $x_{0}$ small enough.

Proof. (partial). We only consider the first assertion under the significantly simplifying hypothesis that $A+B$ is self-adjoint on $D=D_{A} \cap D_{B}$. The second assertion is proven along the same lines.

Let $\psi \in D$ and consider

$s^{-1}\left(e^{i s A} e^{i s B}-I\right) \psi=s^{-1}\left(e^{i s A}-I I\right) \psi+s^{-1} e^{i s A}\left(e^{i s B}-I\right) \psi \rightarrow i A \psi+i B \psi$

as $s \rightarrow 0$ and

$$
s^{-1}\left(e^{i s(A+B)}-I\right) \psi \rightarrow i(A+B) \psi \text { as } s \rightarrow 0 .
$$

Thus, setting $K(s)=s^{-1}\left(e^{i s A} e^{i s B}-e^{i s(A+B)}\right)$, the vector $K(s) \psi \rightarrow 0$ as $s \rightarrow 0$ or $s \rightarrow \infty$, for any $\psi \in D$. Due to the assumed self-adjointness of $A+B$ on $D, A+B$ is closed so that $D$ equipped with the norm

$$
\|\psi\|_{A+B}=\|\psi\|+\|(A+B) \psi\|
$$

is a Banach space. As $K(s): D \rightarrow \mathcal{H}$ is bounded for each finite $s$ and tends to zero strongly at 0 and $\infty$, we can apply the uniform boundedness principle or Banach-Steinhaus Theorem ${ }^{\mathrm{d}}$ to deduce the existence of a constant $C$ so that

$$
\|K(s) \psi\| \leq C\|\psi\|_{A+B}, \quad \forall s \in \mathbb{R}, \quad \forall \psi \in D .
$$

Therefore, on any compact set of $D$ in the $\|\cdot\|_{A+B}$ norm, $K(s) \psi \rightarrow 0$ uniformly as $s \rightarrow 0$. We know from Theorem 5.1 that $e^{i s(A+B)}: D \rightarrow D$ and is strongly continuous, thus $\left\{e^{i s(A+B)} \psi \mid s \in[-1,1]\right\}$ is compact in $D$ for $\|\cdot\|_{A+B}$, for $\psi$ fixed.

Hence

$$
t^{-1}\left(e^{i t A} e^{i t B}-e^{i t(A+B)}\right) e^{i s(A+B)} \psi \rightarrow 0
$$

uniformly in $s \in[-1,1]$ as $t \rightarrow 0$. Therefore, writing

$$
\begin{aligned}
& \left(\left(e^{i t A / n} e^{i t B / n}\right)^{n}-\left(e^{i t(A+B) / n}\right)^{n}\right) \psi= \\
& \sum_{k=0}^{n-1}\left(e^{i t A / n} e^{i t B / n}\right)^{k}\left[e^{i t A / n} e^{i t B / n}-e^{i t(A+B) / n}\right]\left(e^{i t(A+B) / n}\right)^{n-1-k} \psi,
\end{aligned}
$$

we get that the RHS is bounded in norm by

$$
|t| \max _{|s|<t}\left\|\left(\frac{t}{n}\right)^{-1}\left[e^{i t A / n} e^{i t B / n}-e^{i t(A+B) / n}\right] e^{i s(A+B)} \psi\right\| .
$$

\footnotetext{
${ }^{\mathrm{d}}$ If $\mathcal{X}$ and $\mathcal{Y}$ are two Banach spaces, and $\mathcal{F}$ is a family of bounded linear operators from $\mathcal{X}$ to $\mathcal{Y}$ such that for each $x \in \mathcal{X},\left\{\|T x\|_{\mathcal{Y}} \mid T \in \mathcal{F}\right\}$ is bounded, then $\{\|T\| \mid T \in$ $\mathcal{F}\}$ is bounded.
} 
Thus, if $\psi \in D$, we get

$$
\left(e^{i t A / n} e^{i t B / n}\right)^{n} \psi \rightarrow e^{i t(A+B)} \psi, \quad \text { as } n \rightarrow \infty .
$$

The operators being bounded and $D$ being dense, this finishes the proof.

\section{One-Parameter Semigroups}

This Section extends some results of the previous one in the sense that unitary groups discussed above are a particular case of semigroups. The setting used in this Section is that of a Banach space denoted by $\mathcal{B}$. One parameter semigroups will be used in the study the time evolution of Open Quantum Systems.

Definition 6.1. Let $\{S(t)\}_{t \geq 0}$ be a family of bounded operators defined on $\mathcal{B}$. We say that $\{S(t)\}_{t \geq 0}$ is a strongly continuous semigroup or $C_{0}$ semigroup if

1) $S(0)=I I$

2) $S(t+s)=S(t) S(s)$ for any $s, t \geq 0$

3) $S(t) \varphi$ is continuous as a function of $t$ on $[0, \infty)$, for all $\varphi \in \mathcal{B}$.

Remark 6.1.3) is equivalent to requiring continuity at $0^{+}$only.

Definition 6.2. The infinitesimal generator of the semigroup $\{S(t)\}_{t \geq 0}$, is the linear operator $(A, D)$ defined by

$$
\begin{aligned}
& D=\left\{\varphi \in \mathcal{B} \mid \lim _{t \rightarrow 0^{+}} t^{-1}(S(t)-I) \varphi \text { exists in } \mathcal{B}\right\} \\
& A \varphi=\lim _{t \rightarrow 0^{+}} \frac{(S(t)-I I) \varphi}{t}, \varphi \in D .
\end{aligned}
$$

The main properties of semigroups and their generators are listed below.

Proposition 6.1. Let $\{S(t)\}_{t \geq 0}$ be a semigroup of generator A. Then

a) There exist $\omega \in \mathbb{R}$ and $M \geq 1$ such that $\|S(t)\| \leq M e^{\omega t}$, for all $t \geq 0$.

b) The generator $A$ is closed with dense domain $D$.

c) For any $t \geq 0, \varphi \in D$, we have $\int_{0}^{t} S(\tau) \varphi d \tau \in D$ and

$$
A\left(\int_{0}^{t} S(\tau) d \tau\right)=S(t) \varphi-\varphi
$$

d) For any $t \geq 0, S(t): D \rightarrow D$ and if $\varphi \in D, t \mapsto S(t) \varphi$ is in $C^{1}([0, \infty))$ and

$$
\frac{d}{d t} S(t) \varphi=A S(t) \varphi=S(t) A \varphi, \quad t \geq 0 .
$$

e) If $\left\{S_{1}(t)\right\}_{t \geq 0}$ and $\left\{S_{2}(t)\right\}_{t \geq 0}$ are two $C_{0}$ semigroups with the same generator $A$, then $\bar{S}_{1}(t) \equiv S_{2}(t)$. 
Proof. a) By the right continuity at 0 and the Banach-Steinhaus Theorem, there exists $\epsilon>0$ and $M \geq 1$ such that $\|S(t)\| \leq M$ if $t \in[0, \epsilon]$. For any given $t \geq 0$, there exists $n \in \mathbb{N}$ and $0 \leq \delta<\epsilon$ such that $t=n \epsilon+\delta$, hence

$$
\|S(t)\|=\left\|S(\delta) S(\epsilon)^{n}\right\| \leq M^{n+1} \leq M M^{t / \epsilon} \equiv M e^{\omega t}, \text { with } \omega=\frac{\ln M}{\epsilon} \geq 0 .
$$

c) For $\varphi \in D, t \geq 0$ and any $\epsilon>0$,

$$
\begin{aligned}
\frac{(S(\epsilon)-I) \varphi}{\epsilon} \int_{0}^{t} S(\tau) \varphi & =\frac{1}{\epsilon} \int_{0}^{t}(S(\tau+\epsilon)-S(\tau)) \varphi d \tau \\
& =\frac{1}{\epsilon} \int_{t}^{t+\epsilon} S(\tau) \varphi-\frac{1}{\epsilon} \int_{0}^{\epsilon} S(\tau) \varphi
\end{aligned}
$$

which converges to $S(t) \varphi-\varphi$ as $\epsilon \rightarrow 0$.

d) For $\varphi \in D, t \geq 0$ and any $\epsilon>0$, we have

$$
\frac{(S(\epsilon)-I) \varphi}{\epsilon} S(t) \varphi=S(t) \frac{(S(\epsilon)-I) \varphi}{\epsilon} \varphi \rightarrow S(t) A \varphi, \text { as } \quad \epsilon \rightarrow 0 .
$$

Thus $S(t) \varphi \in D$ and $A S(t)=S(t) A$ on $D$. As a consequence of (24), the function $t \mapsto S(t) \varphi$ has a right derivative given by $S(t) A \varphi$ which is continuous on $[0, \infty)$. Therefore, a classical result of analysis shows that the derivative at $t \geq 0$ exists.

b) Let $\varphi$ and define $\varphi_{\epsilon}$ for any $\epsilon>0$ by $\varphi_{\epsilon}=\frac{1}{\epsilon} \int_{0}^{\epsilon} S(\tau) \varphi d \tau$. The vector $\varphi_{\epsilon} \in D$, by c) and $\varphi_{\epsilon} \rightarrow \varphi$ and $\epsilon \rightarrow 0$, so that $D$ is dense. Closedness of $A$ is shown as follows. Let $\left\{\varphi_{n}\right\}_{n \in \mathbb{N}}$ be a sequence of vectors in $D$, such that $\varphi_{n} \rightarrow \varphi$ and $A \varphi_{n} \rightarrow \psi$, for some $\varphi$ and $\psi$. For any $n \in \mathbb{N}$ and $t>0, \mathrm{~d}$ ) implies by integration

$$
S(t) \varphi_{n}-\varphi_{n}=\int_{0}^{t} S(\tau) A \varphi_{n} d \tau
$$

Taking limits $n \rightarrow \infty$ we get $S(t) \varphi-\varphi=\int_{0}^{t} S(\tau) \psi d \tau$, therefore

$$
\lim _{t \rightarrow 0^{+}} t^{-1} S(t) \varphi-\varphi=\psi
$$

In other words, $\psi \in D$ and $\psi=A \varphi$.

e) Finally, for $\varphi \in D, t>0$, we define, for $\tau \in[0, T], \psi(\tau)=S_{1}(t-\tau) S_{2}(\tau) \varphi$. In view of $\mathrm{c}$ ) we are allowed to differentiate w.r.t. $\tau$ and we get

$$
\frac{d}{d \tau} \psi(\tau)=-S_{1}(t-\tau) A S_{2}(\tau) \varphi+S_{1}(t-\tau) A S_{2}(\tau) \varphi=0,
$$

hence $\psi(0)=\psi(t)$, i.e. $S_{1}(t) \varphi=S_{2}(t) \varphi$. The density of $D$ concludes the proof. 
Remark 6.2. If $\{S(t)\}_{t \geq 0}$ is a semigroup that is continuous in norm, i.e. such that $\|S(t)-I\| \rightarrow 0$ as $t \rightarrow 0^{+}$, it is not difficult to show that (see e.g. $[\mathrm{P}]$ ) that there exists $A \in \mathcal{L}(\mathcal{B})$ such that $S(t)=e^{A t}$ for any $t \geq 0$.

Definition 6.3. Semigroups characterized by the bound $\|S(t)\| \leq 1$ for all $t \geq 0$ are called contraction semigroups .

Remark 6.3. There is no loss of generality in studying contraction semigroups in the sense that if the semigroup $\{S(t)\}_{t \geq 0}$ satisfies the bound a) in the Proposition above, we can consider the new $C_{0}$ semigroup $S_{1}(t)=e^{-\beta t} S(t)$ satisfying $\left\|S_{1}(t)\right\| \leq M$. At the price of a change of the norm of the Banach space, we can turn it into a contraction semigroup. Let

$$
\begin{aligned}
& \|\varphi \varphi\| \mid=\sup _{t \geq 0}\left\|S_{1}(t) \varphi\right\|, \\
& \text { such that }\|\varphi\| \leq\|\| \varphi\|\leq M\| \varphi \| \text { and }\left\|S_{1}(\tau) \varphi\right\| \leq\|\| \varphi \| .
\end{aligned}
$$

The characterization of generators of $C_{0}$ semigroups is given by the

Theorem 6.1 (Hille Yosida). Let $(A, D)$ be a closed operator with dense domain. The following statements are equivalent:

1) The operator A generates a $C_{0}$ semigroup $\{S(t)\}_{t \geq 0}$ satisfying

$$
\|S(t)\| \leq M e^{\omega t}, \quad t \geq 0
$$

2) The resolvent and resolvent set of $A$ are such that for all $\lambda>\omega$ and all $n \in \mathbb{N}^{*}$

$$
\rho(A) \supset(\omega, \infty) \text { and } \quad\left\|(A-\lambda)^{-n}\right\| \leq \frac{M}{(\lambda-\omega)^{n}}
$$

3) The resolvent and resolvent set of $A$ are such that for all $n \in \mathbb{N}^{*}$

$$
\rho(A) \supset\{\lambda \in \mathbb{C} \mid \Re \lambda>\omega\} \quad \text { and } \quad\left\|(A-\lambda)^{-n}\right\| \leq \frac{M}{(\Re \lambda-\omega)^{n}}, \Re \lambda>\omega .
$$

Remark 6.4. A proof of this Theorem can be found in $[\mathrm{Y}]$ or $[\mathrm{P}]$. We simply note here that $A$ generates of a contraction semigroup, $M=1, \omega=0$, if and only if $\left\|(A-\lambda)^{-1}\right\| \leq \frac{1}{\Re \lambda}, \Re \lambda>0$. This is trivially true if $A=i H$ in a Hilbert space where $H=H^{*}$, as expected. Moreover, in that case, $A$ and $-A$ satisfy the bound, so that we can construct a group, as we already know, generated by $i H$.

Let us specialize a little by considering contraction semigroups on a Hilbert space $\mathcal{H}$. We can characterize their generator by roughly saying that their real part in non-positive.

Definition 6.4. An operator $(A, D)$ on a Hilbert space $\mathcal{H}$ is called dissipative if for any $\varphi \in D$

$$
\langle\varphi \mid A \varphi\rangle+\langle A \varphi \mid \varphi\rangle=2 \Re(\langle\varphi \mid A \varphi\rangle) \leq 0 .
$$


Proposition 6.2. Let $A$ be the generator of a $C_{0}$ semigroup $\{S(t)\}_{t \geq 0}$ on $\mathcal{H}$. Then $\{S(t)\}_{t \geq 0}$ is a contraction semigroup iff $A$ is dissipative.

Proof. Assume $S(t)$ is a contraction semigroup and $\varphi \in D$. Consider $f(t)=$ $\langle S(t) \varphi \mid S(t) \varphi\rangle$. As a function of $t$, it is differentiable and $0 \geq f^{\prime}(0)=\langle\varphi \mid A \varphi\rangle+$ $\langle A \varphi \mid \varphi\rangle$. Conversely, if $A$ is dissipative, as we have $S(t): D \rightarrow D$, we get for any $t \geq 0$,

$$
f^{\prime}(t)=\langle S(t) \varphi \mid S(t) A \varphi\rangle+\langle A S(t) \varphi \mid S(t) \varphi\rangle \leq 0,
$$

so $f(t)$ is monotonically decreasing and $\|S(t) \varphi\| \leq\|\varphi\|$. As $D$ is dense, $\{S(t)\}_{t \geq 0}$ is a contraction semigroup.

Actually, the notion of dissipative operator can be generalized to the Banach space setting. Moreover, it is still true that dissipative operators and generators of $C_{0}$ contraction semigroups are related. This is the content of the Lumer Phillips Theorem stated below.

Let $\mathcal{B}$ be a Banach space and let $\mathcal{B}^{\prime}$ be its dual. The value of $l \in \mathcal{B}^{\prime}$ at $\varphi \in \mathcal{B}$ is denoted by $\langle l, \varphi\rangle \in \mathbb{C}$. Let us define for any $\varphi \in \mathcal{B}$ the duality set $F(\varphi) \subset \mathcal{B}^{\prime}$ by

$$
F(\varphi)=\left\{l \in \mathcal{B}^{\prime} \mid\langle l, \varphi\rangle=\|\varphi\|^{2}=\|l\|^{2}\right\} .
$$

By the Hahn-Banach Theorem, $F(\varphi) \neq \emptyset$ for any $\varphi \in \mathcal{B}$.

Definition 6.5. An operator $(A, D)$ on a Banach space $\mathcal{B}$ is called dissipative if for any $\varphi \in D$, there exists $l \in F(\varphi)$ such that $\Re(\langle l, A \varphi\rangle) \leq 0$.

The following characterization of dissipative operators avoiding direct duality considerations can be found in $[\mathrm{P}]$ :

Proposition 6.3. An operator $(A, D)$ is dissipative if and only if

$$
\|(\lambda I I-A) \varphi\| \geq \lambda\|\varphi\|, \text { for all } \varphi \in D \text { and all } \lambda>0 .
$$

The link between dissipativity and contraction semigroups is provided by the

Theorem 6.2 (Lumer Phillips). Let $(A, D)$ be an operator with dense domain in a Banach space $\mathcal{B}$.

a) If $A$ is dissipative and there exists $\lambda_{0}>0$ such that $\operatorname{Ran}\left(\lambda_{0} I-A\right)=\mathcal{B}$, then $A$ is the generator of a $C_{0}$ contraction semigroup.

b) If $A$ is the generator of a $C_{0}$ contraction semigroup on $\mathcal{B}$, then $R a n(\lambda I I-$ $A)=\mathcal{B}$ for all $\lambda>0$ and $A$ is dissipative.

The proof of this result can be found in $[\mathrm{P}]$, for example.

We close this Section by considerations on the perturbation of semigroups, or more precisely, of their generators. We stick to our Banach space setting for the end of the Section.

We first show that the property of being a generator is stable under perturbation by bounded operators. 
Theorem 6.3. Let $A$ be the generator of a $C_{0}$ semigroup $\{S(t)\}_{t \geq 0}$ on $\mathcal{B}$ which satisfies the bound 1) in Theorem 6.1. Then, if $B$ is a bounded operator, the operator $A+B$ generates a $C_{0}$ semigroup $\{V(t)\}_{t \geq 0}$ that satisfies the same bound with $M \mapsto M$ and $\omega \mapsto \omega+M\|B\|$. Moreover, if $B$ is replaced by $x B$, the semigroup generated by $A+x B$ is an entire function of the variable $x \in \mathbb{C}$.

Proof. If $A+B$ generates a contraction semigroup $V(t)$, it must solve the following differential equation on $D(A+B)=D(A)$

$$
\frac{d}{d t} V(t)=(A+B) V(t) .
$$

Introducing $S(t)$, the solution of that equation must solve

$$
V(t)=S(t)+\int_{0}^{t} S(t-s) B V(s) d s .
$$

Strictly speaking the above integral equation is true on $D(A)$ only, but as $D(A)$ is dense and all operators are bounded, $(25)$ is true on $\mathcal{B}$, as a strong integral. We solve this equation by iteration

$$
V(t)=\sum_{n \geq 0} S_{n}(t)
$$

where

$$
S_{n+1}(t)=\int_{0}^{t} S(t-s) B S_{n}(s) d s, \quad n=0,1, \ldots, \quad \text { and } \quad S_{0}(t)=S(t) .
$$

All integrals are strongly continuous and we have the bounds

$$
\left\|S_{n}(t)\right\| \leq M^{n+1}\|B\|^{n} e^{\omega t} t^{n} / n !
$$

which are proven by an easy induction. The starting estimate is true by hypothesis on $S(t)$. Thus we see that (26) is absolutely convergent and satisfies

$$
\|V(t)\| \leq M e^{(\omega+M\|B\|) t} .
$$

To show that $V(t)$ defined this way is actually generated by $A+B$, we multiply (25) by $e^{-\lambda t}$ and integrate over $[0, \infty]$, assuming $\Re \lambda>\omega+M\|B\|$, to get

$$
\begin{aligned}
r(\lambda) & =\int_{0}^{\infty} e^{-\lambda t} V(t) d t \\
& =\int_{0}^{\infty} e^{-\lambda t} S(t) d t+\left(\int_{0}^{\infty} e^{-\lambda t} S(t) d t\right) B\left(\int_{0}^{\infty} e^{-\lambda t} V(t) d t\right) \\
& =-(A-\lambda)^{-1}-(A-\lambda)^{-1} B r(\lambda) .
\end{aligned}
$$


This yields $(A+B-\lambda) r(\lambda)=-I I$ and, as $\lambda \in \rho(A+B)$ by our choice of $\lambda$, it follows by computations we went through already that $r(\lambda)=-(A+B-\lambda)^{-1}$. We finally use Hille Yoshida Theorem to conclude. For any $k=0,1,2, \cdots$,

$$
\begin{gathered}
\left\|(A+B-\lambda)^{-k-1}\right\|=\frac{1}{k !}\left\|\frac{d^{k}}{d \lambda^{k}} r(\lambda)\right\| \leq \frac{1}{k !} \int_{0}^{\infty} t^{k}\left|e^{-\lambda t}\right|\|V(t)\| d t \\
\leq \frac{M}{k !} \int_{0}^{\infty} t^{k} e^{-(\Re \lambda-\omega-M\|B\|) t} d t=M(\Re \lambda-\omega-M\|B\|)^{-k-1},
\end{gathered}
$$

which shows that the resolvent of $A+B$ satisfies the estimate of the point 3) of Hille Yosida's Theorem.

Finally, we note that $V(t)$ has the form of a converging series in powers of $B$, which proves the last statement.

More general perturbations of generators of semigroups are allowed under the supplementary hypothesis that both the unperturbed generator and the perturbation generate contraction semigroups.

Theorem 6.4. Let $A$ and $B$ be generators of contraction semigroups and assume $B$ is relatively bounded w.r.t. A with relative bound smaller that 1/2. Then, $A+B$ generates a contraction semigroup.

For a proof, see $[\mathrm{K}]$, for example.

\section{References}

[D] E. B. Davies, Spectral Theory and Differential operators, Cambridge University Press, 1995.

[K] T. Kato, Perturbation Theory of Linear Operators, CIM, Springer 1981.

[P] A. Pazy, Semigroups of Linear Operators and Applications to Partial Differential Equations, Springer, 1983.

[RS] M. Reed, B. Simon, Methods of Modern Mathematical Physics, Vol 1-4, Academic Press, 1971-1978.

[Y] K. Yosida, Functional Analysis, CIM, Springer 1980. 